\title{
Effect of fecal microbiota transplantation on neurological restoration in a spinal cord injury mouse model: involvement of brain-gut axis
}

\section{Yingli Jing}

China Rehabilitation Science Institute

\section{Yan Yu}

China Rehabilitation Science Institute

\section{Fan Bai}

China Rehabilitation Science Institute

\section{Limiao Wang}

China Rehabilitation Science Institute

Degang Yang

Chian Rehabilitation Research Center

Chao Zhang

China Rehabilitation Science Institute

Chuan Qin

China Rehabilitation Science Institute

\section{Mingliang Yang}

China Rehabilitation Research Center

\section{Dong Zhang}

Beijing Friendship Hospital

Yanbing Zhu

Beijing Friendship Hospital

Jianjun Li

China Astronautics Standards Institute

Zhiguo Chen ( $\nabla$ chenzhiguo@gmail.com )

Beijing Stomatological Hospital https://orcid.org/0000-0003-1508-510X

\section{Research}

Keywords: fecal microbiota transplantation, neurological function, GI function, gut microbiota, neuroinflammation

Posted Date: April 1st, 2020 
DOI: https://doi.org/10.21203/rs.3.rs-20108/v1

License: (c) (1) This work is licensed under a Creative Commons Attribution 4.0 International License. Read Full License

Version of Record: A version of this preprint was published at Microbiome on March 7th, 2021. See the published version at https://doi.org/10.1186/s40168-021-01007-y. 


\section{Effect of fecal microbiota transplantation on neurological restoration in a spinal} cord injury mouse model: involvement of brain-gut axis

Yingli Jing ${ }^{1,2,3,4^{*}}$, Yan $\mathrm{Yu}^{1,2,3,4^{*}}$, Fan Bai ${ }^{1,2,3,4^{*}}$, Limiao Wang ${ }^{1,2,3,4}$, Degang Yang ${ }^{1,3,4,5,6}$, Chao Zhang ${ }^{1,3,4,5,6}$, Chuan Qin ${ }^{1,3,4,5,6}$, Mingliang Yang ${ }^{1,3,4,5,6}$, Dong Zhang ${ }^{7,8,9}$, Yanbing Zhu ${ }^{7,8,9}$, Jianjun $\operatorname{Li}^{1,2,3,4,5,6 \&}$, Zhiguo Chen ${ }^{4,10 \&}$

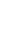

\section{${ }^{1}$ China Rehabilitation Science Institute, Beijing 100068, China}

${ }^{2}$ Institute of Rehabilitation medicine, China Rehabilitation Research Center, Beijing 100068, China

${ }^{3}$ Beijing Key Laboratory of Neural Injury and Rehabilitation, Beijing 100068, China

${ }^{4}$ Center of Neural Injury and Repair, Beijing Institute for Brain Disorders, Beijing 100068, China

${ }^{5}$ Department of Spinal and Neural Function Reconstruction, China Rehabilitation Research Center, Beijing 100068, China

${ }^{6}$ School of Rehabilitation Medicine, Capital Medical University, Beijing 100068,

\section{China}

${ }^{7}$ Immunology Research Center for Oral and Systemic Health, Beijing Friendship Hospital, Capital Medical University, Beijing, 100050, China

${ }^{8}$ Beijing Key Laboratory of Tolerance Induction and Organ Protection in Transplantation, Beijing, 100050, China

${ }^{9}$ Beijing Clinical Research Institute, Beijing, 100050, China

${ }^{10}$ Cell Therapy Center, Beijing Institute of Geriatrics, Xuanwu Hospital Capital 
23

24

25

26

27

28

29

30

31

32

33

34

35

36

37

38

39

40

41

42

43

44

Medical University, National Clinical Research Center for Geriatric Diseases, and Key Laboratory of Neurodegenerative Diseases, Ministry of Education, Beijing 100053, China

* Yingli Jing, Yan Yu and Fan Bai contributed equally to this work.

\& Corresponding authors: Jianjun Li (13718331416@163.com)

and Zhiguo Chen (chenzhiguo@gmail.com. )

Yingli Jing. Email: jingling.1090@163.com.

Yan Yu. Email: yuyancrrc@ 163.com.

Fan Bai. Email: baifan_ren@163.com.

Limiao Wang. Email: limiaowang@foxmail.com.

Degang Yang. Email: yzydg2006@126.com.

Chao Zhang. Email: 790316393@qq.com.

Chuan Qin. Email: qinchuanlove@126.com.

Mingliang Yang. Email: chinayml3@163.com.

Dong Zhang. Email: zhangd@ccmu.edu.cn

Yanbing Zhu. Email:zhuyanbing@ccmu.edu.cn 


\section{Abstract:}

Background: Spinal cord injury (SCI) patients display disruption of gut microbiome and gut dysbiosis exacerbate neurological impairment in SCI models. Cumulative data support an important role of gut microbiome in SCI. Here, we investigated the hypothesis that fecal microbiota transplantation (FMT) may exert a neuroprotective effect on SCI mice.

Results: We found that FMT facilitated functional recovery, promoted neural axonal regeneration, improved animal weight gain and metabolic profiling, and enhanced intestinal barrier integrity and GI motility. High-throughput sequencing revealed that levels of phylum Firmicutes, genus Blautia, Anaerostipes and Lactobacillus were reduced in fecal samples of SCI mice, and FMT remarkably reshaped gut microbiome. Also, FMT-treated SCI mice showed increased amount of fecal short-chain fatty acids (SCFAs), which correlated with alteration of intestinal permeability and locomotor recovery. Furthermore, FMT down-regulated IL-1 $\beta / \mathrm{NF}-\kappa \mathrm{B}$ signaling in spinal cord and NF- $\mathrm{kB}$ signaling in gut.

Conclusion: Our study demonstrates that reprogramming of gut microbiota by FMT improves locomotor and GI functions in SCI mice, possibly through the anti-inflammatory functions of SCFAs.

Keywords: fecal microbiota transplantation, neurological function, GI function, gut microbiota, neuroinflammation 


\section{Background}

The gastrointestinal (GI) tract has the unique property of harboring numerous microbes within the lumen, and the cell number and genetic content outnumber those of the host by a factor of 10 and 150-fold, respectively [1, 2]. The commensal microbiome affects intestinal physiology, and modulates functions of the immune and endocrine systems of host [3-6]. Emerging evidence indicates that the composition and metabolites of microbiome not only regulate normal development in the central nervous system (CNS) such as formation of blood brain barrier, myelination, neurogenesis and microglia maturation [7-9], but also contribute to the onset and/or progression of neurological diseases including Alzheimer's disease, Parkinson's Disease, stroke, depression, anxiety, and autism [10-15].

Traumatic spinal cord injury (SCI) causes neurological impairment and secondary complications [16], such as colorectal, bladder and sexual dysfunctions, among which recovery of bowel function is even prioritized above the ability to walk to some researchers [17-19]. GI dysfunction often manifests as diminished colonic transit, constipation, evacuation dyssynergia, and overflow incontinence, which occur frequently in SCI and may aggravate neurological impairment [20]. Most recently, many lines of evidences demonstrate that aberrant gut microbial community is involved in the pathogenesis and clinical phenotypes of SCI [21-24]. Moreover, a strong correlation between the relative abundance of Clostridiales and Anaeroplasmatales and open-field locomotor has been observed in SCI mice, 
indicating that the proportion of these bacteria might predict the size of functional recovery [22]. Recent investigations demonstrated that induced gut dysbosis exacerbates lesion pathology and impairs functional recovery after SCI; whereas remodeling gut microbes is beneficial to locomotor recovery following injury [25, 23]. These studies have emphasized a strong correlation between gut dysbiosis and SCI, and further imply the significance of gut microbiome in neurological regulation.

Based on fecal transfer experiment and gut microbiota remodeling results, a microbiota-targeted technique, i.e, FMT might be useful for treatment of different CNS diseases. Due to the complexity of gut microbiome and the numerous ways of interaction with host, the mechanisms of FMT in different CNS diseases have not been well understood. Currently, the research field on microbiota-gut-brain axis is gaining more attention in hope of shedding more light on the physiological and pathologic basis of neural restoration. Microbiota and brain communicate via various routes including the immune system and nervous system, involving microbial metabolites such as short-chain fatty acids [26]. In APP/PS1 transgenic mice, FMT alleviates Alzheimer's disease-like pathogenesis by reduction of COX-2 and CD11b levels, and alteration of gut microbiota and SCFAs [27]. In an MPTP-induced Parkinson's disease mouse model, FMT exerts a neuroprotective effect via inhibition of glia cell activation and modulating expression of fecal SCFAs [28]. In this study, we hypothesize that FMT may remodel gut microbiome and be neuroprotective in SCI. 
111

112

113

114

115

116

117

118

119

120

121

122

123

124

125

126

127

\section{Materials}

\section{Animals}

Adult female C57BL/6 (18-22 g) mice were purchased from the Center of Experimental Animals, Capital Medical University (Beijing, China). Mice were kept under standard conditions (temperature $22 \pm 2{ }^{\circ} \mathrm{C}$, humidity $55 \pm 10 \%$ ) with a 12:12 light/dark cycle. Food and water were available ad libitum. Animal protocols have been approved by the Animal Care and Use Committee of Capital Medical University.

\section{Spinal cord injury}

Mice were anesthetized with $2 \%$ isoflurane. After anesthetization, the T10 spinal cord was exposed by laminectomy, followed by a 70-kilodyne contusion using the Infinite Horizons Impactor (Precision Systems \& Instrumentation, Lexington, KY, USA). Afterwards, the muscle and the incision opening were sutured. During the surgical procedure and recovery from anesthesia, mice were placed in a warming chamber until they were completely awake. Postoperatively, animals were hydrated with $0.5 \mathrm{ml}$ Ringer's solution (S. C.) for 5 days. Bladders were voided manually at least twice daily for the duration of the study. Surgical interventions and postoperative animal care were performed in accordance with the guidelines and policies for rodent survival surgery provided by the Experimental Animal Committee of Capital Medical University. 


\section{Experimental groups}

Mice were randomly assigned to four groups (Sham, Sham + FMT, SCI, SCI + FMT) with 20 mice in each group. (1) the Sham group underwent a T10 laminectomy without SCI and received vehicle $(0.1 \mathrm{ml}$ saline $)$; $(2)$ mice in the Sham + FMT group underwent a T10 laminectomy without SCI and were treated with FMT for 4 weeks; (3) mice in the SCI group were subjected to SCI and were given vehicle; and (4) mice in the SCI + FMT group were subjected to SCI and were treated with FMT for 4 weeks. We gave antibiotics to adult mice (6 weeks of age) through drinking water containing $0.2 \mathrm{~g} / \mathrm{L}$ ampicillin, neomycin, and metronidazole, and $0.1 \mathrm{~g} / \mathrm{L}$ vancomycin daily for 2 weeks. After this treatment, a total of $100 \mu$ of the resuspended fecal transplant material was given by oral gavage to FMT mice (Sham+FMT, SCI+FMT) daily over a period of 4 weeks.

\section{Preparation of donor fecal transplant material}

The fecal material was collected and isolated as previously reported [29, 30]. The healthy female C57BL/6 mice were kept in same housing and environmental conditions. Antibiotics untreated, age-matched healthy female mice were used as donors to collect gut microbiota. The donor's fecal pellets were collected under SPF conditions. Stools from donor mice were pooled and $100 \mathrm{mg}$ was re-suspended in 1 $\mathrm{ml}$ of sterile saline (100 mg: $1 \mathrm{ml})$. The solution was vigorously mixed for $10 \mathrm{~s}$ using a benchtop vortex (Vortex-Genie 2, Scientific Industries, USA; speed 9), before centrifugation at $800 \mathrm{~g}$ for $3 \mathrm{~min}$. The supernatant was collected and used as 
155

156

157

158

159

160

161

162

163

164

165

166

167

168

169

170

171

172

173

174

175

176

transplant material as described below. Donor stool was freshly prepared on the day of transplant within $2 \mathrm{~h}$ before oral gavage administration to prevent changes in bacterial composition.

\section{Behavioral analysis}

The Basso Mouse Scale (BMS) was used to score hind limb movements as previously described [31]. Animals were assessed in an open field for 4 min before surgery and on day $3,7,14,21$ and 28 post-surgery. The performance of left and right hind limbs was rated separately and averaged to generate BMS scores and subscores. Specific parameters of locomotion were quantified using the DigiGait Image Analysis System $[32,33]$. Mice were trained at a speed of $15 \mathrm{~cm} / \mathrm{s}$ before SCI for 7 days, and then tested at $4 \mathrm{w}$ at a speed of $9 \mathrm{~cm} / \mathrm{s}$. For each test, at least 5 complete step cycles were recorded, and the movement of each paw was analyzed using the Digigait analysis software (Digigait 12.4). Bilateral hind limb motor function was evaluated using a grip strength meter $[34,35]$. Animals holding a grip bar were rapidly pulled away to assess the peak grip force. Seven consecutive trials were performed weekly for $4 \mathrm{w}$ with the highest and lowest values removed to calculate the mean value for each time point.

\section{Recording of motor-evoked potentials (MEPs)}

Animals were anesthetized with pentobarbital sodium (40 mg/kg, i.p.) and two 30-G stimulating electrodes made of stainless steel were placed overlying the left and right 
177

178

179

180

181

182

183

184

185

186

187

188

189

190

191

192

193

194

195

196

197

198

motor cortex. MEP was elicited by transcranial electrical stimulation with a pulse of 1 ms at $7 \mathrm{~mA}$ using a DS3 constant current isolated stimulator (Digitimer). Responses were recorded from the gastrocnemius muscle using $30-\mathrm{G}$ platinum transcutaneous needle electrodes (distance between recording electrodes was $\sim 1 \mathrm{~cm}$ ). Recording electrodes were connected to an active headstage (3110W Headstage; Warner Instruments) and signals amplified using a DP-311 differential amplifier (Warner Instruments). Amplified signal was acquired by the PowerLab 8/30 data-acquisition system (AD Instruments) at a sampling frequency of $20 \mathrm{kHz}$, digitized and stored in computer for analysis.

\section{Immunohistochemistry}

At $4 \mathrm{w}$ after SCI, animals were perfused with $0.1 \mathrm{M} \mathrm{PBS}\left(\mathrm{pH} 7.4,37^{\circ} \mathrm{C}\right.$ ) followed by 4\% (w/v) paraformaldehyde in 0.1 M PBS. Frozen sections of the spinal cord and colonic tissues were prepared at $20 \mu \mathrm{m}$ thickness by using a cryostat microtome (Leica CM 3500, Wetzlar, Germany) and mounted on gelatin-coated glass slides. Sections were equilibrated in $0.1 \mathrm{M}$ Tris-buffered saline for $10 \mathrm{~min}$. After blocking with $10 \%$ normal goat serum in PBS for $1 \mathrm{~h}$, sections of spinal cord were incubated for $1 \mathrm{~h}$ with primary antibodies, including mouse monoclonal anti-NeuN (1:100, Abcam, Cambridge, MA, USA), rabbit polyclonal anti-synapsin (1:100, Abcam, Cambridge, MA, USA), or rabbit polyclonal anti-NF-200 (1:100, Abcam, Cambridge, MA, USA), and sections of colon were incubated for $1 \mathrm{~h}$ with primary antibodies, including rabbit polyclonal anti-ZO-1 (1:100, Abcam, Cambridge, MA, USA), or 
rabbit monoclonal anti-occludin (1:100, Abcam, Cambridge, MA, USA). After primary antibody incubation, slides were rinsed in PBS, followed by incubation with secondary antibodies. The slides were coverslipped with glycerinum-mounting media and examined by using fluorescence microscopy. The relative fluorescence intensity was calculated by using Image Pro Plus7.0 (Media Cybernetics, Silver Spring, MD, USA).

Paraffin sections of the spinal cord and colonic tissues were prepared at $8 \mu \mathrm{m}$ thickness by using a microtome (Leica RM 2235, Wetzlar, Germany) and mounted on gelatin-coated glass slides. Sections were equilibrated in $0.1 \mathrm{M}$ Tris-buffered saline for $10 \mathrm{~min}$. After incubation in $0.3 \%$ hydrogen peroxide for $30 \mathrm{~min}$, the sections were permeabilized with $0.1 \%$ Triton X-100 for 30 min. After blocking with $10 \%$ normal goat serum in PBS for $1 \mathrm{~h}$, sections of spinal cord were incubated for $1 \mathrm{~h}$ with primary antibodies, including rabbit polyclonal anti- TNF $\alpha$ (1:100, Abcam, Cambridge, MA, USA), rabbit polyclonal anti- IL-1 $\beta$ (1:100, Abcam, Cambridge, MA, USA), or rabbit polyclonal anti- NF- $\mathrm{B}$ (1:100, Abcam, Cambridge, MA, USA). After primary antibody incubation, slides were rinsed in PBS, followed by incubation with secondary antibodies. The slides were coverslipped with glycerinum-mounting media and examined by fluorescence microscopy. The relative gray value was calculated by using Image Pro Plus7.0 (Media Cybernetics, Silver Spring, MD, USA).

\section{Metabolic parameters}


221 Metabolic parameters were measured by using metabolic phenotyping chambers

222 (Mouse Promethion Continuous caging system; Sable Systems ${ }^{\mathrm{TM}}$, Las Vegas, NV).

223 To start the metabolic phenotyping, animals were transferred to the chambers

224 individually under normal housing conditions. Air within the cages was sampled

225 through micro-perforated stainless steel sampling tubes located around the bottom of

226 the cages, above the bedding. Gas sensors were calibrated before each run with $100 \%$

$227 \mathrm{~N}_{2}$ as reference value zero. The incurrent flow rate was set at $2000 \mathrm{~mL} / \mathrm{min}$ and gases

228 were sampled continuously for each cage, from multiple points within the cage (250

$229 \mathrm{ml} / \mathrm{min})$. Oxygen consumption and carbon dioxide $\left(\mathrm{CO}_{2}\right)$ production were recorded

230 for each mouse. Respiratory exchange quotient (RQ) was calculated as the ratio of

$231 \mathrm{CO}_{2}$ production over $\mathrm{O}_{2}$ consumption. Energy expenditure was calculated using the

232 Weir equation: $\mathrm{Kcal} / \mathrm{h}=60 *\left(0.003941 * \mathrm{VO}_{2}+0.001106 * \mathrm{VCO}_{2}\right)$ [36]. Values

233 were calculated after application of algorithms using macros provided with the 234 analysis software ExpeData [37].

\section{FITC-dextran permeability assay}

237 Four weeks after injury, the mice were fasted for $14 \mathrm{~h}$ and gavaged with fluorescein 238 isothiocyanate-dextran (FITC-dextran, 4 KD; Sigma-Aldrich, Madrid, Spain) at a 239 dose of $60 \mathrm{mg}$ per $100 \mathrm{~g}$ body weight in a volume of $0.2 \mathrm{ml}$. Four hours later, the 240 blood were collected by cardiac puncture and clotted for $30 \mathrm{~min}$, followed by a $90 \mathrm{~s}$ 241 centrifugation at 6,000 g. Serum was equally diluted with PBS, and $100 \mu$ dilution was measured in a 96-well plate using a plate reader (EnSpire; Perkin Elmer) at an 
excitation of $481 \mathrm{~nm}$ and an emission of $524 \mathrm{~nm}$. The FITC-dextran was quantified by referring to standard curve measurements in the same plate.

\section{Gastrointestinal transit assessment}

GI motility was assessed by using radiographic methods as described previously at 4 $\mathrm{w}$ following injury. In detail, animals were intragastrically gavaged of barium $(0.2 \mathrm{ml}$, $2 \mathrm{~g} / \mathrm{ml}$ ), and immobilized in a prone position by using an adjustable hand-made transparent plastic tube. The plain facial radiographs of the GI tract were obtained using a digital X-ray apparatus (Siemens; $50 \mathrm{kV}, 10 \mathrm{~mA}$ ) and processed with NPG Real DVD Studio II software. Exposure time was adjusted to $0.06 \mathrm{~s}$. To further reduce stress, mice were released immediately after each image shot (immobilization lasted 1-2 min). X-rays were recorded at different times (immediately and 0.5, 1, 2, 3, 4, 6 and $8 \mathrm{~h}$ after administration of barium). Analysis of the radiographs was performed by a trained investigator blind to the different groups. Alterations in GI motility were semiquantitatively determined from the images by assigning a compounded value to indicated regions of the GI tract considering the following parameters: percentage of the GI region filled with contrast (0-4); intensity of contrast (0-4); homogeneity of contrast (0-2); and sharpness of the GI region profile (0-2). Each of these parameters was scored and a sum (0-12 points) was made. X-ray images were also morphometrically analyzed using ImageJ software (version 1.38, National Institute of Health, USA), and the alterations in the stomach and colorectum were quantified. 


\section{Microbial DNA extraction and PCR amplification}

Microbial DNA was extracted from stool samples using the E.Z.N.A.® Stool DNA Kit (Omega Bio-tek, Norcross, GA, U.S.) according to manufacturer's protocols. The V3-V4 region of the bacteria $16 \mathrm{~S}$ rRNA gene was amplified by using PCR $\left(95^{\circ} \mathrm{C}\right.$ for 2 min, followed by 25 cycles at $95{ }^{\circ} \mathrm{C}$ for $30 \mathrm{~s}, 55^{\circ} \mathrm{C}$ for $30 \mathrm{~s}$, and $72{ }^{\circ} \mathrm{C}$ for $30 \mathrm{~s}$ and a final extension at $72{ }^{\circ} \mathrm{C}$ for $5 \mathrm{~min}$ ) using primers $338 \mathrm{~F} \quad 5^{\prime}$ ACTCCTACGGGAGGCAGCA-3' and 506R 'GGACTACHVGGGTWTCTAAT-3'. PCR reactions were performed in triplicate $20 \mu \mathrm{L}$ mixture containing $4 \mu \mathrm{L}$ of $5 \times$ FastPfu Buffer, $2 \mu \mathrm{L}$ of $2.5 \mathrm{mM}$ dNTPs, $0.8 \mu \mathrm{L}$ of each primer $(5 \mu \mathrm{M}), 0.4 \mu \mathrm{L}$ of FastPfu Polymerase, and $10 \mathrm{ng}$ of template DNA.

\section{Illumina MiSeq sequencing}

Amplicons were extracted from 2\% agarose gels, purified by using the AxyPrep DNA Gel Extraction Kit (Axygen Biosciences, Union City, CA, U.S.), and quantified by using QuantiFluor ${ }^{\mathrm{TM}}$-ST (Promega, U.S.). Purified amplicons were pooled in equimolar and paired-end sequenced $(2 \times 300 \mathrm{bp})$ on an Illumina MiSeq platform according to the standard protocols.

\section{Processing of sequencing data}

Raw fastq files were quality-filtered by Trimmomatic and merged by FLASH with the following criteria: (i) The reads were truncated at any site receiving an average quality score $<20$ over a 50 bp sliding window. (ii) Sequences whose overlap being longer 
than $10 \mathrm{bp}$ were merged according to their overlap with mismatch no more than $2 \mathrm{bp}$.

(iii) Sequences of each sample were separated according to barcodes (exact match) and primers (allowing 2 nucleotide mismatch), and reads containing ambiguous bases were removed.

Operational taxonomic units (OTUs) were clustered with 97\% similarity cutoff using UPARSE (version 7.1) with a novel 'greedy' algorithm that performs chimera filtering and OTU clustering simultaneously. The taxonomy of each 16S rRNA gene sequence was analyzed by RDP Classifier algorithm against the Silva (SSU128) 16S rRNA database using confidence threshold of 70\% [38].

\section{Fecal SCFA detection}

Fecal samples were weighed and then $20 \mathrm{mg}$ transferred into a $2 \mathrm{ml} \mathrm{EP}$ tube, and 1 $\mathrm{mL}$ phosphoric acid $(0.5 \% \mathrm{v} / \mathrm{v})$ solution was added in the EP tube, followed by vortexing for $10 \mathrm{~min}$, and ultrasonic wave treatment for $5 \mathrm{~min}$. Then $0.1 \mathrm{~mL}$ supernatant was added to a $1.5 \mathrm{~mL}$ centrifugal tube, and $0.5 \mathrm{~mL}$ MTBE (containing internal standard) solution was added, followed by vortexing for $3 \mathrm{~min}$, and ultrasound treatment for $5 \mathrm{~min}$. After that, the samples were centrifuged for $10 \mathrm{~min}$ at $12,000 \mathrm{r} / \mathrm{min}$ at $4{ }^{\circ} \mathrm{C}$. After centrifugation, the supernatant was analyzed using a gas chromatography-mass spectrometry (GC-MS/MS 7890B-7000D; Agilent Technologies Inc.) on a silica capillary column (DB-FFAP, $30 \mathrm{~m} \times 0.25 \mathrm{~mm} \times 0.25 \mathrm{um}$, Agilent J\&W) under the following conditions: injected sample size, $2 \mu \mathrm{L}$, splitless; 
injector temperature at $200{ }^{\circ} \mathrm{C}$; initial oven temperature at $95{ }^{\circ} \mathrm{C}$, held for $1 \mathrm{~min}$, raised to $100{ }^{\circ} \mathrm{C}$ at a rate of $25^{\circ} \mathrm{C} / \mathrm{min}$, raised to $130{ }^{\circ} \mathrm{C}$ at a rate of $17{ }^{\circ} \mathrm{C} / \mathrm{min}$, held for $0.4 \mathrm{~min}$, raised to $200^{\circ} \mathrm{C}$ at a rate of $25^{\circ} \mathrm{C} / \mathrm{min}$, held for $0.5 \mathrm{~min}$. Helium was used as a carrier gas at $1.2 \mathrm{ml} / \mathrm{min}$.

The main mass spectrometry conditions were as follows: Ion Source, EI; transfer line temperature, $230{ }^{\circ} \mathrm{C}$; ion source temperature, $230{ }^{\circ} \mathrm{C}$; quad temperature, $150{ }^{\circ} \mathrm{C}$, electron energy, $70 \mathrm{eV}$; scan mode, MRM; and solvent delay, 2.4 min.

\section{Western blot analysis}

A section of spinal cord $1 \mathrm{~cm}$ of length (epicenter $\pm 5 \mathrm{~mm}$ ) and colonic tissue $1 \mathrm{~cm}$ of length were collected. The total protein was prepared in a lysis buffer (Beyotime, China) by lysing tissue homogenates for $1 \mathrm{~h}$, and then centrifuged at 14,000 $\mathrm{g}$ for 8 min at $4{ }^{\circ} \mathrm{C}$. The protein content of the supernatant was determined by using a protein assay kit (BCA, Pierce, Rockford, IN, USA). Equal amounts of total protein (50 $\mu \mathrm{g})$ were separated by using $12 \%$ sodium dodecyl sulfate-polyacrylamide gel electrophoresis and transferred to polyvinylidene difluoride membranes. The membranes were blocked with $5 \%$ non-fat skim milk in Tris-buffered saline solution with $0.05 \%$ Tween-20 (TBST) for $1 \mathrm{~h}$, and then incubated with antibodies against

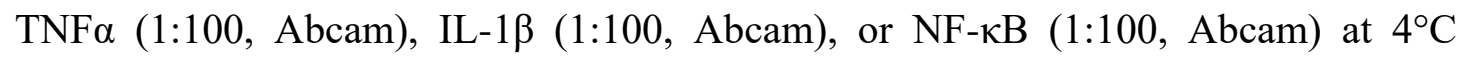
overnight. After washing 3 times with TBST, appropriate horseradish peroxidase-conjugated secondary antibodies were added. $\beta$-actin $(1: 1000$, Cell 
Signaling Technology) was used as an internal control. The bands were visualized by using enhanced chemiluminescence, and images were acquired with ChemiDoc MP System (Bio-Rad, Hercules, CA, USA). The relative band intensities were quantified by using Quantity One (Bio-Rad, Hercules, CA, USA).

\section{Statistical analysis}

Results were presented as the mean values with the standard error of mean (SEM).

The data were analyzed by using SPSS, version 17.0 statistic software package (SPSS Inc., Chicago, Illinois, USA). Student's t-tests were used to determine significance between two groups. Multivariate analysis of variance (MANOVA) was conducted to test between-group differences on the dependent measures. One-way analysis of variance followed by post-hoc Tukey's analysis was performed to compare groups of three or more. In addition, relationships between significant SCFAs changes and behavior scores (BMS), BMS subscores and gut permeability were evaluated using the Pearson correlation method. All analyses were conducted with an alpha level of $p$ $<0.05$ using SPSS statistical software. Effect size was reported as either a R2 for the correlation analysis or Partial Eta2 for MANOVA.

Qiime software package (Version 1.7.0) was used to analyze alpha diversity and beta diversity. Alpha diversity indices referring to community richness (Ace and Chao) were calculated based on the OTUs information of Sham, Sham+FMT, SCI and SCI+FMT groups. The beta diversities, including principal co-ordinate analysis 
353

(PCoA) and non-metric multidimensional scaling (NMDA), were evaluated with uniFrac-weighted distance created by Qiime 1.7.0. Differential abundance of genera was tested by using Wilcoxon rank sum test, and $\mathrm{P}$ values were corrected for multiple testing with Benjamin and false discovery rate method.

\section{Results}

\section{FMT treatment improves locomotor recovery in spinal cord injury mice.}

The locomotor recovery was observed during the four weeks post-injury in all groups. FMT treatment further significantly increased hind limb locomotor function starting from fourteen days after injury compared to that of SCI group, and the improvement in BMS scores and BMS subscores continued until the end of the experiment (Fig. 1a-b). Moreover, digigait and grip analysis are complementary behavioral tests used to evaluate the hindlimb gait and strength. The percentage of stride duration spent in the swing phase was decreased in SCI mice and this parameter was significantly improved in FMT treatment group (Fig. 1c). The percentage of the stride duration spent in the stance phase was significantly reduced following FMT treatment compared to SCI group (Fig. 1c). Accordingly, FMT treatment resulted in a significant increased swing to stance ratio in SCI mice (Fig. 1c). Additionally, stride length was increased and stride frequency was decreased, which was reversed by FMT treatment (Fig. 1d). To a certain extent, FMT were able to ameliorate the gait abnormality induced by SCI, and increase the coordination of gait. Measurements of grip strength were presented as a percentage of the baseline grip strength. All animals demonstrated 
a gradual continued recovery of grip strength after injury. After treatment, grip strength differed between SCI group and FMT group showing a nonsignificant trend of improvement at 7 day post injury (dpi), 14 dpi and 21 dpi. Only at 28 dpi, FMT treatment group demonstrated a statistically significant improvement compared to SCI group (Fig. 1e). Altogether, mice treated with FMT exhibited a relatively greater locomotor recovery compared to injured mice without FMT treatment.

\section{FMT treatment facilitates restoration of descending motor pathways.}

Next, to examine the function of descending pathways from the motor cortex to the hindlimb motor neurons, we monitored motor evoked potentials (MEPs) at $4 \mathrm{w}$ after injury (Fig. 2). MEPs, which reflect the connectivity of neuromuscular unit, were recorded at the gastrocnemius muscle after electrical stimulation on the motor cortex (Fig. 2a). In Sham and Sham+FMT groups, typical waveforms of MEPs were detected. Following injury, the MEPs were mostly abolished, indicating a disruption of the neuromuscular unit. With FMT treatment, the SCI mice displayed larger amplitude, compared to SCI mice without FMT treatment (Fig. 2b). The quantification of the amplitudes in different groups was shown in Figure 2c. Additionally, SCI mice developed significant delayed (to $17.8 \mathrm{~ms}$ ) in MEP latency compared with Sham group (4.63 ms), the latencies of MEP showed no significant difference between SCI and SCI+FMT groups, although there was a trend toward shorter latencies in the FMT group (Fig. 2d). These observations indicated that FMT treatment is beneficial, and may further enhance functional recovery after SCI.

\section{FMT treatment promotes neuronal survival and synaptic regeneration.}


To investigate the anatomical basis of the observed locomotor recovery, we performed immunofluorescent staining on the spinal cord sections (Fig. 3). In the Sham group, neurons in the ventral horn appeared normal, with large cell bodies and intact axons. SCI induced a significant loss of NeuN-positive neurons. However, the number of NeuN-positive cells was significantly higher in the FMT group compared to that of the SCI group (Fig. 3a). The quantification of neuronal cell bodies across the four groups was shown in Figure 3b. Synapsin (SYN) is a type of vesicle protein that marks the presynaptic membranes. Immunostaining analysis showed a reduction in synapsin staining in SCI group at $4 \mathrm{w}$ post-injury, and the staining intensity was increased by FMT treatment (Fig. 3a, 3c). Neurofilaments (NF-200) are cell type-specific proteins abundant in neuronal axons and the staining has been applied for evaluation of neuronal and axonal damage [39, 40]. Compared with the SCI group, staining against NF-200 in the lesion areas was increased with FMT treatment at $4 \mathrm{w}$ post-injury (Fig. 3a, 3d). The data suggested that FMT treatment may have promoted neuronal survival and axonal regeneration after traumatic SCI.

\section{FMT treatment improves weight gain and metabolic profile in SCI mice.}

The body weights were monitored and compared at indicated time points. The Sham and Sham+FMT groups showed stable and comparable body weights over the $4 \mathrm{w}$ of observation. For both SCI and SCI+FMT groups, body weight rapidly decreased during the first 3 days after surgery, and gradually gained back in the following days. Compared with SCI group, SCI+FMT mice gained more weight, which showed 
statistically significant difference at 14, 21 and 28 days post-injury (Fig. 4a). Consistent with these results, an increase in food intake and water consumption was observed in the SCI+FMT group as compared to the SCI group, especially at day 28 post-injury (Fig. 4b-c). In addition, the metabolic parameters were assessed over a 24 h period at week 4 post-injury. Indirect calorimetry revealed that the average energy expenditure was significantly elevated with FMT treatment in SCI animals (Fig. 4d-e). As shown in Figure 4f and g, the average respiratory exchange quotient (RQ) values were restored in SCI+FMT group. These data demonstrated that FMT treatment after SCI led to more food and water consumption, higher energy expenditure, and greater body weight gains.

\section{FMT treatment is conducive to maintaining intestinal barrier integrity in SCI} mice.

Altered intestinal barrier integrity and subsequent gastrointestinal dysfunction have been postulated as a pathophysiological event in SCI. To test whether FMT treatment had any impact on intestinal barrier permeability after SCI, the mice were gavaged with FITC-labeled dextran (4 KD) at week 4 post-injury, and FITC levels in blood were measured. Permeability in colon was more severe in SCI mice compared to that of SCI+FMT mice (Fig. 5a). Intestinal tight junctions (TJs) have been shown to be associated with intestinal barrier integrity [41]. Therefore, we investigated the expression and distribution of TJ proteins in the colon. As depicted in Figure 5c, expressions of ZO-1 and occludin indicated an increased disruption and 
disorganization at the apical surface, and FMT treatment stabilized TJ structures, as evidenced by smooth and organized localization of ZO-1 and occludin (Fig. 5b). The results demonstrated that FMT treatment had facilitated to maintain intestinal barrier integrity and upregulate the expression of tight junction proteins after SCI.

\section{FMT treatment accelerates GI transit in SCI mice.}

GI transit, as an overall measure of GI motility, was assessed in mice by using barium gavage followed by X-ray imaging. Representative images from different groups were shown in Fig. 6a at different time points (2, 3 and 8 h). Overall, SCI mice displayed a higher level of filling of GI tract, indicative of a slower GI transit, while FMT treatment accelerated GI transit in mice after injury (Fig. 6a). With regard to filling of colorectum, the dynamics for Sham+FMT group was similar to that for Sham group.

GI motility was markedly delayed after injury, which was improved at certain time points in SCI+FMT mice (Fig. 6a-b). Morphometric analysis showed that injury increased the filling of colorectum, which was reversed by FMT treatment (Fig. 6a, 6c). Collectively, FMT treatment might have contributed to the faster GI transit following SCI.

\section{FMT treatment modulates gut microbiota composition in SCI mice.}

To test whether FMT has modulated gut microbiota, we performed 16S rRNA (V3+V4 regions) gene sequencing to analyze the bacterial taxonomic composition following microbial therapy in SCI mice. Based on a 97\% similarity threshold, the 
effective reads were clustered into 221 operational taxonomic units (OTUs), which included 123 species, 90 genera, 46 families, 30 orders, 20 classes and 11 phyla. As shown in Figure $7 \mathrm{a}$ and $7 \mathrm{~b}$, there were significant differences in the ace and chao indices between the sham group and SCI group. FMT treatment significantly increased the richness of the intestinal microbiota in SCI mice. To measure the degree of similarity between microbial communities, $\beta$ diversity was further evaluated by using uniFrac-weighted principal coordinate analysis ( $\mathrm{PCoA}$ ) and non-metric multi-dimensional scaling (NMDS). Sham+FMT group clustered closely to Sham group, suggesting that FMT treatment did not have significant effect on gut microbiota in normal animals without SCI. SCI group clustered distinctly from sham group, and FMT treatment changed the profile of SCI-disrupted gut microbiota (Fig. 7c-d). The differences in microbial communities among groups suggested that the presence of gut dysbiosis in SCI mice was modulated following FMT treatment. By investigating the abundance and distribution of gut microbiota in Sham group, SCI group and SCI+FMT group, we identified a number of altered microbiota that might be responsible for microbial dysbiosis. The heatmap showed a significant difference in the relative abundance across the groups at the phylum level (Fig. 7e). Compared to Sham group, the abundance of Firmicutes was reduced in SCI group, which was reversed by FMT treatment. In contrast, the abundance of Bacteroidetes in SCI mice did not differ from those of Sham and SCI+FMT mice (Fig. 7e). To further explore the changes in the gut microbial community structure among groups, the genus level analysis was performed. As shown in Figure 7f, SCI significantly decreased the 
relative abundance of Blautia, Anaerostipes and Lactobacillus. FMT treatment markedly increased the relative abundance of Blautia and Anaerostipes, while it had little effect on that of Lactobacillus (Fig. 7f). Compared to Sham group, Sham+FMT group did not show significant changes in microbiota at phylum and genus levels (data not shown). Together, these data indicated that FMT treatment could have modulated the microbiota composition to alleviate gut microbial dysbiosis in SCI mice.

\section{FMT treatment restores fecal short-chain fatty acids (SCFAs) in SCI mice}

Certain end products of fermentation by the gut microbes could enter the bloodstream and impact the physiology of the CNS in the host [7, 42]. Among the potential factors regulating the microbiota gut-brain axis, microbial metabolites SCFAs may be the major mediators. We examined the fecal concentrations of certain SCFAs including acetic acid, propionic acid, butyric acid, isobutyric acid, valeric acid, isovaleric acid and caproic acid in the different groups. Among the tested fecal SCFAs, butyric acid content was altered to the greatest extent, a decrease by $58.6 \%$ in SCI compared to that of sham group. FMT upregulated fecal butyric acid level by $46.7 \%$ compared to that of SCI mice (Fig. 8b). Similar to butyric acid, propionic acid and isobutyric acid were also decreased in SCI mice by $21.2 \%$ and $39.4 \%$, respectively, compared to those of Sham group, and FMT treatment significantly augmented fecal propionic acid and isobutyric acid levels by $21.3 \%$ and $65.0 \%$ in SCI mice (Fig. 8a, 8c). Caproic acid was decreased by $31.8 \%$ in SCI mice, but FMT treatment did not significantly 
change the amount of caproic acid (Fig. 8d). Additionally, no significant changes were found as to acetic acid, valeric acid and isovaleric acid (Table s1). The data suggested that FMT treatment might have mediated pathophysiological changes in the host through alteration of fecal SCFAs.

\section{Correlations between SCFAs and locomotor recovery/ intestinal barrier} permeability.

We next assessed whether the contents of propionic acid, butyric acid, and isobutyric acid were correlated with locomotor recovery or intestinal integrity. A multivariate analysis of variance (MANOVA) was performed with groups as the independent variable, motor outcomes (BMS scores and subscores), intestinal permeability (FITC-dextran) and level of metabolies (Butyricacid, Propionicacid, Isobutyricacid) as the dependent variables. Hotelling's Trace revealed a significant multivariate effect of group on corresponding index $[\mathrm{F}(12,30)=33.83 ; \mathrm{p}<0.001]$. Univariate ANOVAs revealed significant effects among different groups on BMS scores $[F(2,21)=97.04$; $\mathrm{p}<0.001]$, BMS Subscore $[\mathrm{F}(2,21)=210.79 ; \mathrm{p}<0.001]$, FITC-dextran $[\mathrm{F}(2,21)=$ 10.04; $\mathrm{p}=0.001]$, Butyricacid $[\mathrm{F}(2,21)=7.79 ; \mathrm{p}=0.003]$, Propionicacid $[\mathrm{F}(2,21)=$ 4.76; $\mathrm{p}=0.02]$ and Isobutyricacid $[\mathrm{F}(2,21)=3.61 ; \mathrm{p}=0.045]$ (Table $\mathrm{s} 2)$.

As shown in Figure 9, the content of propionic acid was positively correlated with open field locomotor (BMS) scores and BMS subscores; similar results were obtained with regard to the correlation of butyric acid amount to BMS scores and BMS 
subscores. Inverse relationships were found between the content of butyric acid and FITC-dextran permeability. In addition, the content of isobutyric acid was positively correlated with BMS subscores. And the association between the content of isobutyric acid and BMS scores / FITC-dextran permeability was not statistically significant. It seems that the contents of certain SCFAs could provide certain predictive power as to functional recovery and barrier integrity after SCI.

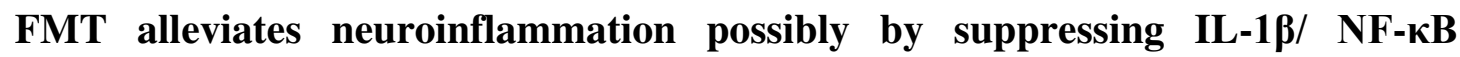
signaling pathway.

To further explore the molecular interactions between gut microbial dysbiosis and neuroinflammation in SCI, we characterized spinal cord expression of TNF $\alpha$, IL-1 $\beta$ and NF- $\kappa \mathrm{B}$ by applying immunohistochemical analysis and western blotting. Figure $10 \mathrm{a}$ and $\mathrm{b}$ indicated that the expression of TNF $\alpha$ did not show significant alterations among different groups. While, a substantial increase in IL- $1 \beta$ and NF- $\kappa B$ positive staining was found in spinal cord tissues collected from mice at $4 \mathrm{w}$ after SCI. However, treatment with FMT reduced the positive staining of both IL-1 $\beta$ and NF- $\kappa B$. Next, the result of western blotting indicated that FMT treatment remarkably attenuated the expressions of IL- $1 \beta$ and NF- $\kappa$ B following injury and did not significantly alter the expression of TNF $\alpha$, which was consistent with the result of immunostaining analysis.

FMT alleviates gut inflammation possibly by suppressing NF-кB signaling 


\section{pathway.}

We also investigated whether inflammation was involved in gut. Figure $11 \mathrm{a}$ and $\mathrm{b}$ showed that the expression of TNF $\alpha$ and IL-1 $\beta$ did not differ significantly between groups. However, expression of NF- $\mathrm{BB}$ was upregulated in the colon of SCI mice vs. that of Sham mice, and SCI+FMT mice down-regulated NF-кB pathway in colon

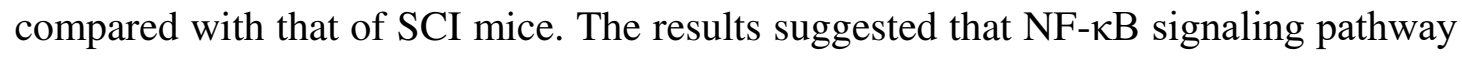
was involved in gut inflammation and neuroinflammation. Recent studies have indicated that SCFAs exert anti-inflammatory and neuroprotective effects in neurodegenerative disorders [28, 43]. FMT may alter SCFA expression profiles and alleviate gut inflammation and neuroinflammation.

\section{Discussion}

Accumulating evidence suggests that gut microbial dysbiosis may be involved in SCI pathogenesis and clinical manifestations $[22,23,25]$. In the present study, we found that FMT not only reshaped microbiota in SCI mice, but also improved neurological functions of SCI mice. FMT could attenuate GI dysfunction and modulate microbiota metabolites in SCI mice. Moreover, FMT had no apparent side effect on behavioral and GI functions in sham mice. These data suggest that FMT treatment can ameliorate gut microbial dysbiosis and modulate metabolites, which subsequently lead to alleviated functional impairment, elevated neuromuscular connection and improved neurological regeneration.

Studies have shown that gut microbial dysbiosis occurs in SCI patients and 
murine models of SCI. Kigerl and colleagues reported that microbiome dysbiosis impedes functional recovery in SCI mice, which can be reversed by administration of probiotics [23]. In the previous study we explored the functions of melatonin on intestinal microbiota in SCI mice. That study showed that gut microbiota may have directly or indirectly contributed to melatonin-induced beneficial effects in SCI mice [25]. In the current study, we continued to investigate whether FMT can be applied as an interventional approach to improve functional recovery in SCI; and if so, what components of the fecal transplant might have exerted such effects. By employing a mouse SCI model, we revealed that phylum Firmicutes, genus Blautia and genus Anaerostipes may be involved in SCI-induced dysbiosis and act as the effective components in fecal transplant.

Through 16S rRNA sequencing, we identified Firmicutes and Bacteroidetes (two major bacterial taxa) that were changed in SCI mice, and FMT treatment significantly increased the amount of Firmicutes. A positive correlation was observed between the level of Firmicutes and locomotor recovery. However, phylum Firmicutes consists of a mixture of members with different, even opposing effects; therefore it is required to investigate further down to the genus level, which revealed that Blautia and Anaerostipes levels were reversed by FMT treatment. These microbes belong to the putative short-chain fatty acid (SCFA)-producing bacteria and butyrate-producing bacteria, respectively. Studies have showed that SCFA-producing bacteria may benefit the host through protecting the mucosa from pathogen-induced damage, 
supplying nutrients to colonocytes, and mitigation of inflammation [44, 45]. The interactions between the resident beneficial gut bacteria (eg SCFA-producing bacteria) and opportunistic pathogens (eg endotoxin-producing bacteria) have been considered as a crucial factor for intestinal homeostasis [46]. Decrease of SCFA-producing bacteria has been observed as a quite common phenomenon in metabolic diseases such as insulin resistance and obesity [46, 47], two indications that frequently occured in SCI patients. A previous study showed that certain drug intervention (e.g. berberin) can effectively prevent high-fat diet (HFD)-induced insulin resistance through enriching SCFA-producing Blautia and Allobaculum in the gut of rats [47]. Liping Zhao and colleagues reported that, enrichment of SCFA-producing bacteria by administration of berberine or metformin may be beneficial to ameliorate HFD-induced obesity in rats [48]. In the current study, increase of the genus Blautia and Anaerostipes was observed following FMT, indicating that enrichment of SCFA-producing bacteria may be a potential mechanism underlying the beneficial effect of FMT treatment.

SCFAs are usually produced by gut commensal bacteria through anaerobic fermentation of undigested carbohydrates. These particular fatty acids are relatively small in size, thus are capable of crossing the blood brain barrier (BBB) via monocarboxylate transporters to impact the physiology of the CNS [7, 42, 49]. SCFAs have profound effect on gut function by mediating intestinal immune function and suppressing intestinal inflammation [50-52]. Also, SCFAs have potent 
619

620

621

622

623

624

625

626

627

628

629

630

631

632

633

634

635

636

637

638

639

640

anti-inflammatory effects on macrophages and can suppress ongoing inflammation in the CNS $[53,54]$. Among the SCFAs, sodium butyrate exerts neuroprotective effects and anti-inflammatory properties following spinal cord injury [43]. Kukkar et al. showed that oral administration of butyrate attenuates neuropathic pain symptoms in a chronic constriction injury (CCI) model, which may be mainly attributed to its ability to reduce the release of proinflammatory mediators during neuropathy development [56]. Clinical data revealed a significant reduction in butyrate producing phylum members in SCI patients, suggesting that reduced levels of butyrate may have an impact on long-term recovery after SCI [21]. Our study demonstrates that the decreased amount of fecal SCFAs in SCI mice correlates with higher levels of inflammation in spinal cord and colon. Accordingly, we observed a higher amount of fecal SCFAs and a lower level of inflammatory indicators such as IL-1 $\beta$ and NF- $\kappa$ B in spinal cord and NF- $\mathrm{BB}$ in colon, following FMT treatment in SCI mice. The study suggests that FMT may exert a neuroprotective effect in SCI via upregulating expression of fecal SCFAs and suppressing ongoing inflammation.

However, some concerns existed regarding the application of FMT for SCI treatment. The foremost concern is the safety of FMT, although most studies have shown that adverse events associated with FMT are mild and transient, such as abdominal discomfort, nausea, vomiting, bloating or flatulence [56, 57]. Recent studies reported unpredictable risk of drug-resistant E.coli Bacteremia transmitted by FMT $[58,59]$ and FDA has since issued safety warning regarding clinical application 
641

642

643

644

645

646

647

of FMT. In our study, no obvious side effects of FMT were observed on sham group, but more thorough investigations on the safety and adverse reactions are necessary prior to clinical use can be considered. Another issue is related to the complexity of gut microbiome itself and the numerous ways it may interact with the host. Most microbiota studies rely on 16S RNA sequencing technology, which still lacks the resolution power to go beyond Genus level. Therefore, it would be difficult to identify microbes at strain level and delineate the causative relationship that may lead to a more precise interventional strategy. But this issue may be solved with advancement of sequencing technology and optimization of data processing [60]. Another new approach in gut microbiota studies is application of bacteriophages, which seems to have the power to modulate gut microbiota at a strain level [61]. Future studies may take advantage of these new technologies to further elucidate the mechanisms underlying the impact of FMT on SCI mice, and to tease out the causative relationship of microbiota-host and microbiota-microbiota interactions.

\section{Conclusions}

FMT treatment facilitated functional recovery, promoted neural axonal regeneration, improved animal weight gain and metabolic profiling, as well as enhanced intestinal barrier integrity and GI motility. Additionally, FMT treatment significantly altered the composition of intestinal microbiota and the amount of fecal short-chain fatty acids. Furthermore, FMT down-regulated IL-1 $\beta / \mathrm{NF}-\mathrm{\kappa B}$ signaling in spinal cord and NF- $\mathrm{KB}$ signaling in gut. The dat demonstrated that FMT reprogramming gut microbiota 
663

664

665

666

667

668

669

670

671

672

673

674

675

676

677

678

679

680

681

682

683

684

improved locomotor function and GI function which might concentrate to the anti-inflammatory of SCFAs in SCI mice.

\section{Declarations}

\section{Acknowledgements}

Not applicable.

\section{Availability of data and materials}

All data generated or analysed during this study are included in this published article and its supplementary information file.

\section{Funding}

This work was supported by the National Natural Science Foundation of China (81901272) and the Special Fund for Basic Scientific Research of Central Public Research Institutes, grant number: 2016cz-1, 2018cz-8.

\section{Authors' contributions}

JL and ZC conceived the study and directed the study; YJ, YY and FB designed the experiments, interpreted the results and wrote the manuscript; LW and DY carried out the animal experiments and obtained the data; YJ, CZ and CQ performed behavioral tests, biochemical experiments and histological analysis; YJ and FB analyzed the data; MY, DZ and YZ revised the manuscript. All authors have read and approved the final version of the manuscript.

\section{Competing interests}

The authors declare that they have no competing interests. 
685

686

687

688

689

690

691

692

693

694 1. de Vos WM, de Vos EA. Role of the intestinal microbiome in health and disease:

695

696 2. Lozupone CA, Stombaugh JI, Gordon JI, Jansson JK, Knight R. Diversity,

697

698 3. Simren M, Barbara G, Flint HJ, Spiegel BM, Spiller RC, Vanner S, et al.

699 Intestinal microbiota in functional bowel disorders: a Rome foundation report.

700

701

702

703 5. Round JL, Mazmanian SK. The gut microbiota shapes intestinal immune

704

705

706

\section{Consent for publication}

All the authors consented to the publication of the study once it is accepted.

\section{Ethics approval and consent to participate}

Animal protocols have been approved by the Animal Care and Use Committee of Capital Medical University. Surgical interventions and postoperative animal care were performed in accordance with the guidelines and policies for rodent survival surgery provided by the Experimental Animal Committee of Capital Medical University.

\section{References} from correlation to causation. Nutr Rev. 2012;70 Suppl 1:S45-56. stability and resilience of the human gut microbiota. Nature. 2012;489:220-30. Gut. 2013;62:159-76.

4. Clemente JC, Ursell LK, Parfrey LW, Knight R. The impact of the gut microbiota on human health: an integrative view. Cell. 2012;148:1258-70.

responses during health and disease. Nat Rev Immunol. 2009;9:313-23.

6. Forsythe P, Kunze WA. Voices from within: gut microbes and the CNS. Cell Mol Life Sci. 2013;70:55-69. 
707 7. Sharon G, Sampson TR, Geschwind DH, Mazmanian SK. The Central Nervous $708 \quad$ System and the Gut Microbiome. Cell. 2016;167:915-32.

709 8. Hoban AE, Stilling RM, Ryan FJ, Shanahan F, Dinan TG, Claesson MJ, et al.

710 Regulation of prefrontal cortex myelination by the microbiota. Transl Psychiatry.

$711 \quad 2016 ; 6: \mathrm{e} 774$.

712 9. Braniste V, Al-Asmakh M, Kowal C, Anuar F, Abbaspour A, Toth M, et al. The 713 gut microbiota influences blood-brain barrier permeability in mice. Sci Transl $714 \quad$ Med. 2014;6:263ra158.

715 10. Liu P, Wu L, Peng G, Han Y, Tang R, Ge J, et al. Altered microbiomes distinguish 716 Alzheimer's disease from amnestic mild cognitive impairment and health in a 717 Chinese cohort. Brain Behav Immun. 2019;80:633-43.

718 11. Sampson TR, Debelius JW, Thron T, Janssen S, Shastri GG, Ilhan ZE, et al. Gut 719 Microbiota Regulate Motor Deficits and Neuroinflammation in a Model of $720 \quad$ Parkinson's Disease. Cell. 2016;167:1469-80 e12.

721 12. Benakis C, Brea D, Caballero S, Faraco G, Moore J, Murphy M, et al. 722 Commensal microbiota affects ischemic stroke outcome by regulating intestinal 723 gammadelta T cells. Nat Med. 2016;22:516-23.

724 13. Foster J, Neufeld KA. Gut-brain axis: How the microbiome influences anxiety 725 and depression. Trends Neurosci. 2013;36:305-12.

726 14. Kang DW, Adams JB, Gregory AC, Borody T, Chittick L, Fasano A, et al. 727 Microbiota Transfer Therapy alters gut ecosystem and improves gastrointestinal and autism symptoms: an open-label study. Microbiome. 2017;5. 
729 15. Chen K, Luan X, Liu Q, Wang J, Chang X, Snijders AM, et al. Drosophila 730 Histone Demethylase KDM5 Regulates Social Behavior through Immune Control $731 \quad$ and Gut Microbiota Maintenance. Cell Host Microbe. 2019;25:537-52 e8.

732 16. Bauman WA, Spungen AM. Carbohydrate and lipid metabolism in chronic spinal $733 \quad$ cord injury. J Spinal Cord Med. 2001;24:266-77.

734 17. Lynch AC, Antony A, Dobbs BR, Frizelle FA. Bowel dysfunction following $735 \quad$ spinal cord injury. Spinal Cord. 2001;39:193-203.

736 18. Anderson KD. Targeting recovery: priorities of the spinal cord-injured population. $737 \quad$ J Neurotrauma. 2004;21:1371-83.

738 19. Simpson LA, Eng JJ, Hsieh JT, Wolfe DL, Spinal Cord Injury Rehabilitation 739 Evidence Scire Research T. The health and life priorities of individuals with $740 \quad$ spinal cord injury: a systematic review. J Neurotrauma. 2012;29:1548-55.

741 20. White AR, Holmes GM. Anatomical and Functional Changes to the Colonic $742 \quad$ Neuromuscular Compartment after Experimental Spinal Cord Injury. J $743 \quad$ Neurotrauma. 2018;35:1079-90.

744 21. Gungor B, Adiguzel E, Gursel I, Yilmaz B, Gursel M. Intestinal Microbiota in $745 \quad$ Patients with Spinal Cord Injury. PLoS One. 2016;11:e0145878.

746 22. Zhang C, Zhang W, Zhang J, Jing Y, Yang M, Du L, et al. Gut microbiota 747 dysbiosis in male patients with chronic traumatic complete spinal cord injury. $\mathbf{J}$ $748 \quad$ Transl Med. 2018;16:353.

749 23. Kigerl KA, Hall JCE, Wang LL, Mo XK, Yu ZT, Popovich PG. Gut dysbiosis 750 impairs recovery after spinal cord injury. J Exp Med. 2016;213:2603-20. 
751 24. O'Connor G, Jeffrey E, Madorma D, Marcillo A, Abreu MT, Deo SK, et al. 752 Investigation of Microbiota Alterations and Intestinal Inflammation Post-Spinal 753 Cord Injury in Rat Model. J Neurotrauma. 2018;35:2159-66.

754 25. Jing YL, Yang DG, Bai F, Zhang C, Qin C, Li D, et al. Melatonin Treatment 755 Alleviates Spinal Cord Injury-Induced Gut Dysbiosis in Mice. J Neurotrauma. $756 \quad 2019 ; 36: 2646-64$.

757 26. Cryan JF, O'Riordan KJ, Cowan CSM, Sandhu KV, Bastiaanssen TFS, Boehme $758 \quad$ M, et al. The Microbiota-Gut-Brain Axis. Physiol Rev. 2019;99:1877-2013.

759 27. Sun J, Xu J, Ling Y, Wang F, Gong T, Yang C, et al. Fecal microbiota 760 transplantation alleviated Alzheimer's disease-like pathogenesis in APP/PS1 $761 \quad$ transgenic mice. Transl Psychiatry. 2019;9:189.

762 28. Sun MF, Zhu YL, Zhou ZL, Jia XB, Xu YD, Yang Q, et al. Neuroprotective 763 effects of fecal microbiota transplantation on MPTP-induced Parkinson's disease 764 mice: Gut microbiota, glial reaction and TLR4/TNF-alpha signaling pathway. 765 Brain Behav Immun. 2018;70:48-60.

766 29. Chang CJ, Lin CS, Lu CC, Martel J, Ko YF, Ojcius DM, et al. Ganoderma 767 lucidum reduces obesity in mice by modulating the composition of the gut 768 microbiota. Nat Commun. 2015;6:7489.

769 30. Borody TJ, Paramsothy S, Agrawal G. Fecal microbiota transplantation: 770 indications, methods, evidence, and future directions. Curr Gastroenterol Rep. $771 \quad 2013 ; 15: 337$.

772 31. Basso DM, Fisher LC, Anderson AJ, Jakeman LB, McTigue DM, Popovich PG. 
Basso Mouse Scale for locomotion detects differences in recovery after spinal cord injury in five common mouse strains. J Neurotrauma. 2006;23:635-59.

775 32. Sashindranath M, Daglas M, Medcalf RL. Evaluation of gait impairment in mice 776 subjected to craniotomy and traumatic brain injury. Behav Brain Res.

777 $2015 ; 286: 33-8$.

778 33. Suzuki H, Ahuja CS, Salewski RP, Li LJ, Satkunendrarajah K, Nagoshi N, et al. 779 Neural stem cell mediated recovery is enhanced by Chondroitinase ABC 780 pretreatment in chronic cervical spinal cord injury. Plos One. 2017;12:e0182339.

781 34. Iwasaki M, Wilcox JT, Nishimura Y, Zweckberger K, Suzuki H, Wang J, et al. Synergistic effects of self-assembling peptide and neural stem/progenitor cells to 783 promote tissue repair and forelimb functional recovery in cervical spinal cord $784 \quad$ injury. Biomaterials. 2014;35:2617-29.

785 35. Onifer SM, Rodriguez JF, Santiago DI, Benitez JC, Kim DT, Brunschwig JP, et al. 786 Cervical spinal cord injury in the adult rat: assessment of forelimb dysfunction. 787 Restor Neurol Neurosci. 1997;11:211-23.

788 36. Weir JB. New methods for calculating metabolic rate with special reference to 789 protein metabolism. J Physiol. 1949;109:1-9.

790 37. Kothari V, Luo Y, Tornabene T, O'Neill AM, Greene MW, Geetha T, et al. High 791 fat diet induces brain insulin resistance and cognitive impairment in mice.

792 Biochim Biophys Acta Mol Basis Dis. 2017;1863:499-508.

793 38. Amato KR, Yeoman CJ, Kent A, Righini N, Carbonero F, Estrada A, et al. Habitat 794 degradation impacts black howler monkey (Alouatta pigra) gastrointestinal 
microbiomes. ISME J. 2013Jul;7:1344-53.

796 39. Petzold A. Neurofilament phosphoforms: surrogate markers for axonal injury, 797 degeneration and loss. J Neurol Sci. 2005;233:183-98.

798 40. Posmantur R, Hayes RL, Dixon CE, Taft WC. Neurofilament 68 and 799 neurofilament 200 protein levels decrease after traumatic brain injury. J $800 \quad$ Neurotrauma. 1994;11:533-45.

801 41. Grander C, Adolph TE, Wieser V, Lowe P, Wrzosek L, Gyongyosi B, et al. 802 Recovery of ethanol-induced Akkermansia muciniphila depletion ameliorates 803 alcoholic liver disease. Gut. 2018;67:891-901.

804 42. Mitchell RW, On NH, Del Bigio MR, Miller DW, Hatch GM. Fatty acid transport 805 protein expression in human brain and potential role in fatty acid transport across 806 human brain microvessel endothelial cells. J Neurochem. 2011;117:735-46.

807 43. Lanza M, Campolo M, Casili G, Filippone A, Paterniti I, Cuzzocrea S, et al. 808 Sodium Butyrate Exerts Neuroprotective Effects in Spinal Cord Injury. Mol $809 \quad$ Neurobiol. 2019;56:3937-47.

810 44. Maslowski KM, Vieira AT, Ng A, Kranich J, Sierro F, Yu D, et al. Regulation of 811 inflammatory responses by gut microbiota and chemoattractant receptor GPR43. $812 \quad$ Nature. 2009;461:1282-6.

813 45. De Filippo C, Cavalieri D, Di Paola M, Ramazzotti M, Poullet JB, Massart S, et 814 al. Impact of diet in shaping gut microbiota revealed by a comparative study in 815 children from Europe and rural Africa. Proc Natl Acad Sci U S A. 2010;107:14691-6. 
817 46. Qin J, Li Y, Cai Z, Li S, Zhu J, Zhang F, et al. A metagenome-wide association 818 study of gut microbiota in type 2 diabetes. Nature. 2012;490:55-60.

819 47. Zhang X, Zhao Y, Zhang M, Pang X, Xu J, Kang C, et al. Structural changes of 820 gut microbiota during berberine-mediated prevention of obesity and insulin 821 resistance in high-fat diet-fed rats. PLoS One. 2012;7(8):e42529.

822 48. Zhang X, Zhao Y, Xu J, Xue Z, Zhang M, Pang X, et al. Modulation of gut 823 microbiota by berberine and metformin during the treatment of high-fat 824 diet-induced obesity in rats. Sci Rep. 2015;5:14405.

82549 . Vijay N, Morris ME. Role of monocarboxylate transporters in drug delivery to 826 the brain. Curr Pharm Des. 2014;20:1487-98.

827 50. Arpaia N, Campbell C, Fan X, Dikiy S, van der Veeken J, deRoos P, et al. 828 Metabolites produced by commensal bacteria promote peripheral regulatory 829 T-cell generation. Nature. 2013;504:451-5.

830 51. Smith PM, Howitt MR, Panikov N, Michaud M, Gallini CA, Bohlooly YM, et al. 831 The microbial metabolites, short-chain fatty acids, regulate colonic Treg cell 832 homeostasis. Science. 2013;341:569-73.

833 52. Sun J, Chang EB. Exploring gut microbes in human health and disease: Pushing 834 the envelope. Genes Dis. 2014;1:132-9.

835 53. Chen PS, Wang CC, Bortner CD, Peng GS, Wu X, Pang H, et al. Valproic acid 836 and other histone deacetylase inhibitors induce microglial apoptosis and attenuate 837 lipopolysaccharide-induced dopaminergic neurotoxicity. Neuroscience. 2007;149:203-12. 
839 54. Kim HJ, Rowe M, Ren M, Hong JS, Chen PS, Chuang DM. Histone deacetylase 840 inhibitors exhibit anti-inflammatory and neuroprotective effects in a rat 841 permanent ischemic model of stroke: multiple mechanisms of action. J Pharmacol $842 \quad$ Exp Ther. 2007;321:892-901.

843 55. Kukkar A, Singh N, Jaggi AS. Attenuation of neuropathic pain by sodium 844 butyrate in an experimental model of chronic constriction injury in rats. J Formos $845 \quad$ Med Assoc. 2014;113:921-8.

846 56. Wang JW, Kuo CH, Kuo FC, Wang YK, Hsu WH, Yu FJ, et al. Fecal microbiota 847 transplantation: Review and update. J Formos Med Assoc. 2019;118 Suppl $848 \quad 1: S 23-S 31$.

849 57. Vindigni SM, Surawicz CM. Fecal Microbiota Transplantation. Gastroenterol $850 \quad$ Clin North Am. 2017;46:171-85.

851 58. Blaser MJ. Fecal Microbiota Transplantation for Dysbiosis - Predictable Risks. N $852 \quad$ Engl J Med. 2019;381:2064-6.

853 59. DeFilipp Z, Bloom PP, Torres Soto M, Mansour MK, Sater MRA, Huntley MH, 854 et al. Drug-Resistant E. coli Bacteremia Transmitted by Fecal Microbiota 855 Transplant. N Engl J Med. 2019;381:2043-50.

856 60. Zou Y, Xue W, Luo G, Deng Z, Qin P, Guo R, et al. 1,520 reference genomes 857 from cultivated human gut bacteria enable functional microbiome analyses. Nat $858 \quad$ Biotechnol. 2019;37:179-85.

859 61. Hsu BB, Gibson TE, Yeliseyev V, Liu Q, Lyon L, Bry L, et al. Dynamic 860 Modulation of the Gut Microbiota and Metabolome by Bacteriophages in a 


\section{Figure legend}

Fig. 1 Effect of FMT treatment on locomotor recovery. a, b Time course of locomotor analysis by using an automated treadmill (DigiGait). (c) Swing to stride ratio, stance to stride ratio, and swing to stance ratio. (d) Stride length and stride frequency. e Hindlimb grip strength. Data were normalized to pretreatment (post-injury) baseline with a value " 1 " referring to no difference after treatment and values above "1" indicating improvement. $* \mathrm{p}<0.05$ compared to SCI group, ${ }^{* *} \mathrm{p}<0.01$ compared to SCI group. LR, left rear; RR: right rear.

Fig. 2 Effect of FMT treatment on spinal cord conduction capability. a A schematic diagram of MEP recording experiment. MEP was recorded from the gastrocnemius muscle in an anesthetized state after electrical stimulation on the motor cortex. b Representative MEPs recorded from Sham mice and SCI mice that had received the

877 indicated treatment $4 \mathrm{w}$ after injury. The amplitude (c) and the latency (d) were quantified and statistically analyzed. ${ }^{*} \mathrm{p}<0.05$ compared to Sham group; $* * \mathrm{p}<0.01$ compared to Sham group; \#\#p<0.01 compared to SCI group.

880

Fig. 3 Effect of FMT treatment on neuronal survival and synaptic regeneration 882 following SCI. a NeuN-positive neurons were examined in the different treatment groups by immunofluorescence staining $(n=4)$. b Quantification of NeuN-positive 
884

885

886

887

888

889

890

891

892

893

894

895

896

897

898

899

900

901

902

903

904

905

neuronal cell bodies in the T10 region in different groups. a,c Quantification of synapsin immunoreactivity (red) with representative images of the ventral horn. a,d Quantification of NF-200 immunoreactivity (red) with representative images of the ventral horn. Scale bar, $50 \mu \mathrm{m} .{ }^{*} \mathrm{p}<0.05$ compared to Sham group; $* * \mathrm{p}<0.01$ compared to Sham group; \#p<0.05 compared to SCI group; \#\#p<0.01 compared to SCI group.

Fig. 4 Effect of FMT treatment on body weight, food intake, water consumption and metabolism. a Changes in body weight over time in Sham, Sham+FMT, SCI, and SCI+FMT groups. b-c food intake (b) and water consumption (c) were examined during the 4 weeks in the four groups. Respiratory quotient (RQ) (d) and energy expenditure (EE) (f) were measured at the end of the experiments. The mean respiratory quotient (Avg_RQ) (e) and mean energy expenditure (Avg_EE) (g) were measured every $5 \mathrm{~min}$ for $24 \mathrm{~h}$ in $\mathrm{SCI}$ group and SCI+FMT group. ${ }^{*} \mathrm{p}<0.05$ compared to SCI group; $* * p<0.01$ compared to SCI group.

Fig. 5 Effect of FMT treatment on intestinal permeability and expression of tight junction proteins. a Intestinal permeability was assessed 4 weeks following injury by measuring FITC intensity in serum after oral gavage of FITC-dextran. b-c Quantification of occludin immunoreactivity or ZO-1 immunoreactivity (green) with representative immunofluorescence images of colon sections. DAPI, blue; $* \mathrm{p}<0.05$ compared to SCI group; Scale bar, $50 \mu \mathrm{m} ;{ }^{* *} \mathrm{p}<0.01$ compared to SCI group. 
907 Fig. 6 Effect of FMT on gastrointestinal motility in SCI mice. a Representative 908 images of Sham, Sham+FMT, SCI, and SCI+FMT groups at 2, 3 and $8 \mathrm{~h}$ after 909 administration of barium. b Filling of colorectum was measured by radiological 910 methods. c Colorectum size was determined by using Image J. **p<0.01 compared to 911 Sham group; \#p<0.05 compared to SCI group; \#\#p<0.01 compared to SCI group.

Fig. 7 Effect of FMT treatment on gut bacterial composition after SCI. a-b Comparison of ace index (a) and chao index (b) based on OUT levels in the four groups. c-d Scatter plots of principal coordinate analysis (PCoA) (c) and non-metric multi-dimensional scaling (NMDS) (d) showing similarity of the bacterial communities based on uniFrac-weighted distance. (e) Bacterial composition of the different communities at phylum level and quantitative analyses of relative abundances of Firmicutes and Bacteroidetes among different groups. (f) Bacterial composition of different communities at genus level and quantitative analyses of the relative abundances of Blautia, Anaerostipes and Lactobacillus among different groups. $* \mathrm{p}<0.05$ compared to Sham group; $* * \mathrm{p}<0.01$ compared to Sham group; $\# \mathrm{p}<0.05$ compared to SCI group.

Fig. 8 Effect of FMT treatment on fecal SCFA levels in mice. The SCI-mediated decrease of propionic acid, butyric acid and isobutyric acid expression was ameliorated by FMT treatment. a Fecal propionic acid content. b Fecal butyric acid 
content. c Fecal isobutyric acid content. The SCI-mediated decrease of caproic acid expression was not significantly changed by FMT treatment. d Fecal caproic acid content. SCFAs were analyzed by GC-MS. *p<0.05 compared to SCI group; **p<0.01 compared to SCI group; NS, not significant.

Fig. 9 Correlations between SCFAs and BMS scores/BMS subscores/FITC-dextran permeability. A-c Correlations between propionic acid and BMS scores (a), BMS subscores (b), and FITC-dextran permeability (c). d-f Correlations between butyric acid and BMS scores (d), BMS subscores (e), and FITC-dextran permeability (f). h-g Correlations between isobutyric acid and BMS scores (h), BMS subscores (i), and FITC-dextran permeability (g).

Fig. 10 Effect of FMT treatment on expression of TNF $\alpha$, IL- $1 \beta$ and NF- $\kappa B$ in spinal cord. a TNF $\alpha$, IL- $1 \beta$ and NF- $\kappa \mathrm{B}$ were stained by immunofluorescence on spinal cord sections from each group. Scale bar, $50 \mu \mathrm{m}$. b The expression of TNF $\alpha$, IL-1 $1 \beta$ and NF- $\kappa \mathrm{B}$ was detected by western blot, and the relative amount of TNF $\alpha$ (c), IL-1 $\beta$ (d) and NF- $\kappa \mathrm{B}(\mathbf{e})$ were semi-quantified.

Fig. 11 Effect of FMT treatment on expression of TNF $\alpha$, IL-1 $\beta$ and NF- $\kappa B$ in colon. a TNF $\alpha$, IL- $1 \beta$ and NF- $\kappa$ B were stained by immunofluorescence on colonic tissue from each group. Scale bar, $50 \mu \mathrm{m}$. b The expression of TNF $\alpha$, IL-1 $\beta$ and NF- $\kappa B$ were analyzed by western blot, and the relative amount of TNF $\alpha(\mathbf{c}), \operatorname{IL}-1 \beta$ (d) and 
$950 \mathrm{NF}-\kappa \mathrm{B}(\mathbf{e})$ were semi-quantified in the different treatment groups.

951

952 
Figures

$\mathbf{a}$

$\rightarrow \mathrm{SCl}$

$\rightarrow \mathrm{SCl}+\mathrm{FMT}$

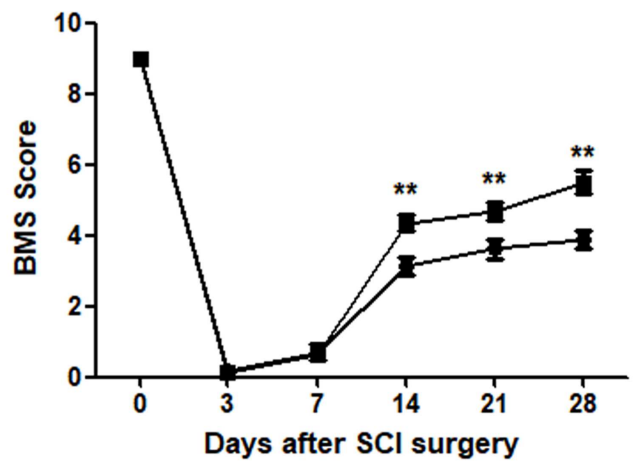

b

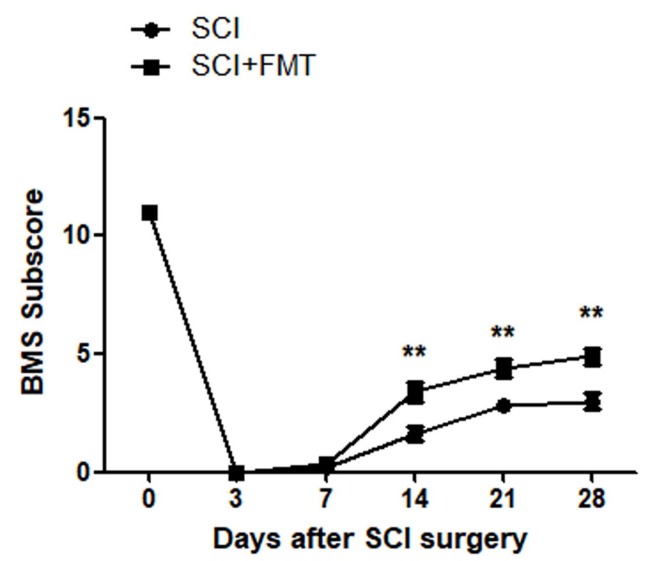

c $\quad \mathrm{msCl}_{\mathrm{SCl}+\mathrm{FMT}}$

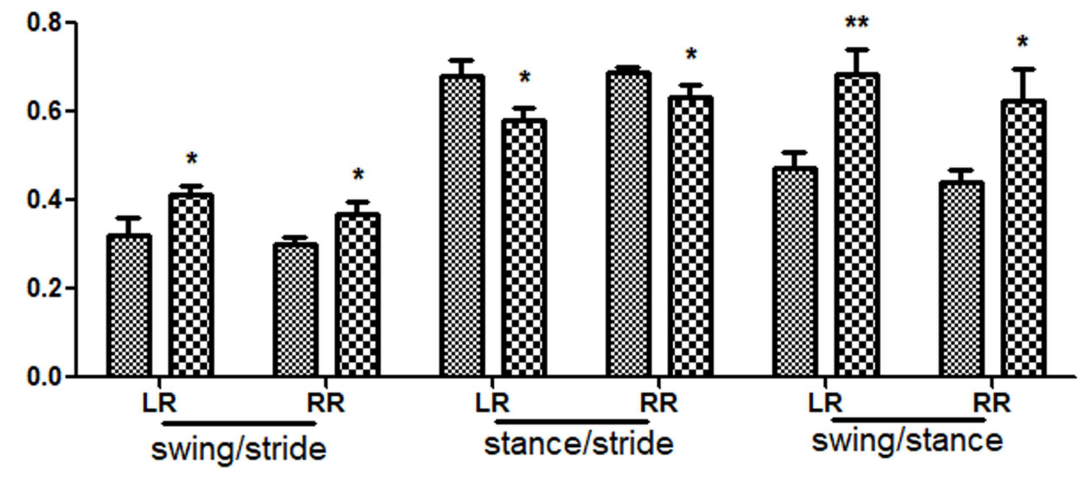

d $\mathrm{sCl}$

$\infty \mathrm{SCl}+\mathrm{FMT}$

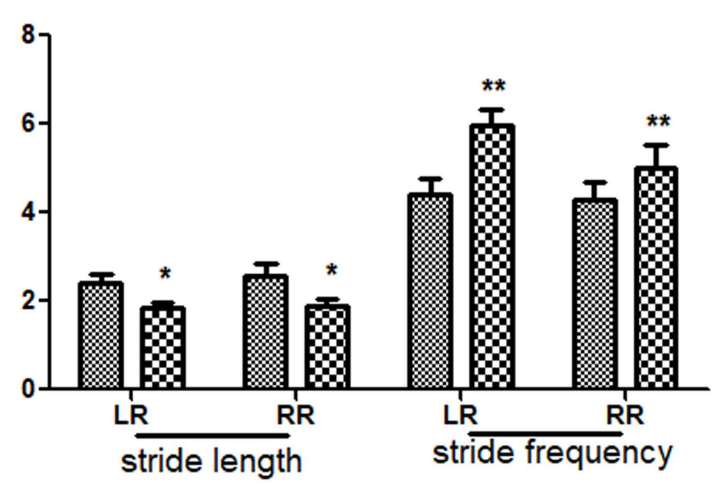

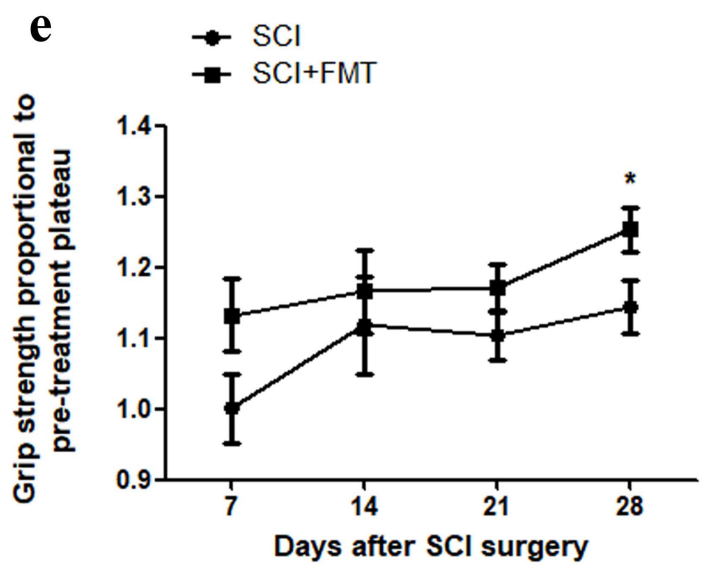

Figure 1

Effect of FMT treatment on locomotor recovery. a, b Time course of locomotor functional recovery as assessed by BMS (a) and BMS subscore (b). c-d Gait analysis by using an automated treadmill (DigiGait). (c) Swing to stride ratio, stance to stride ratio, and swing to stance ratio. (d) Stride length and 
stride frequency. e Hindlimb grip strength. Data were normalized to pretreatment (post-injury) baseline with a value " 1 " referring to no difference after treatment and values above " 1 " indicating improvement. ${ }^{*} \mathrm{p}<0.05$ compared to $\mathrm{SCl}$ group, ${ }^{* *} \mathrm{p}<0.01$ compared to $\mathrm{SCl}$

A
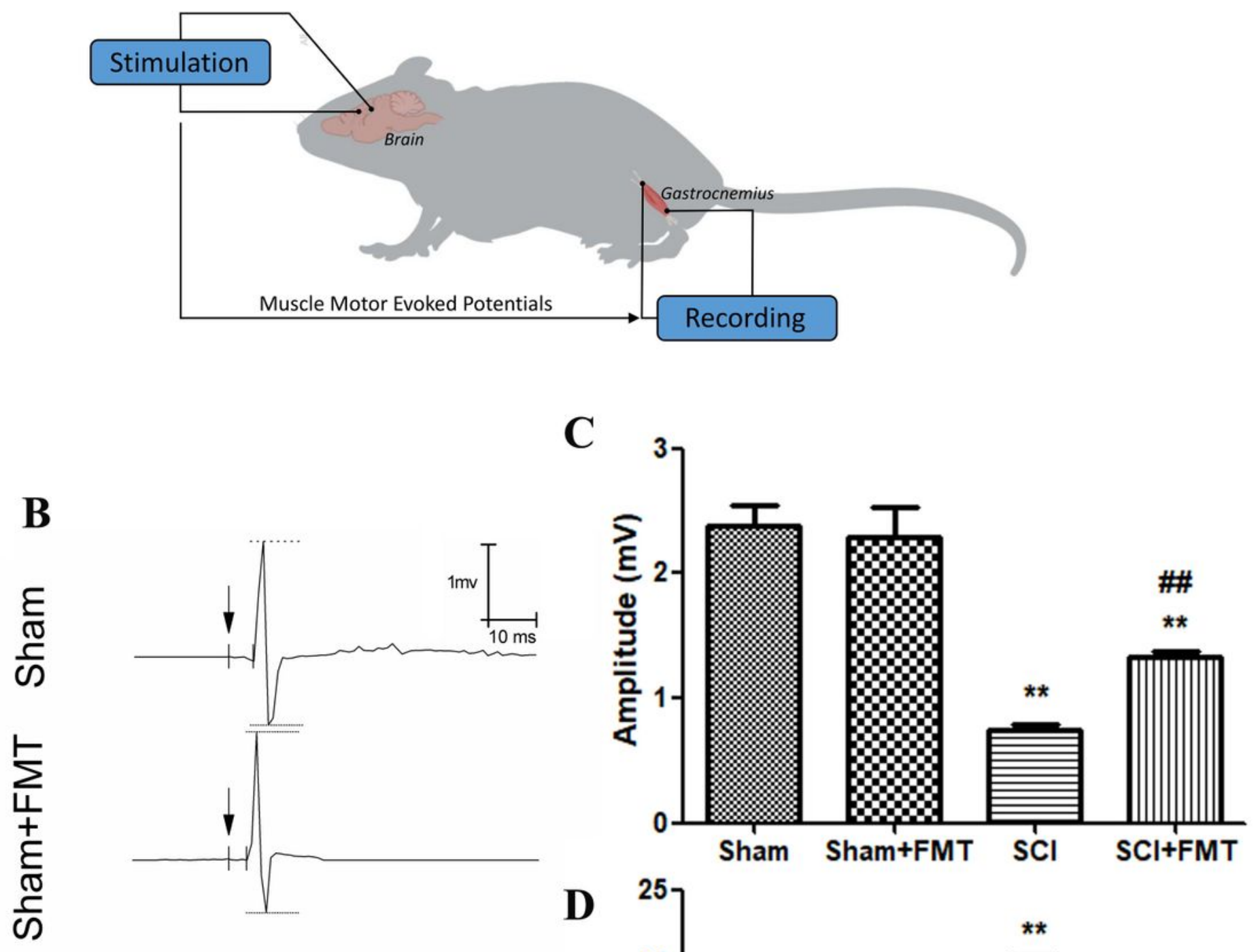

ত
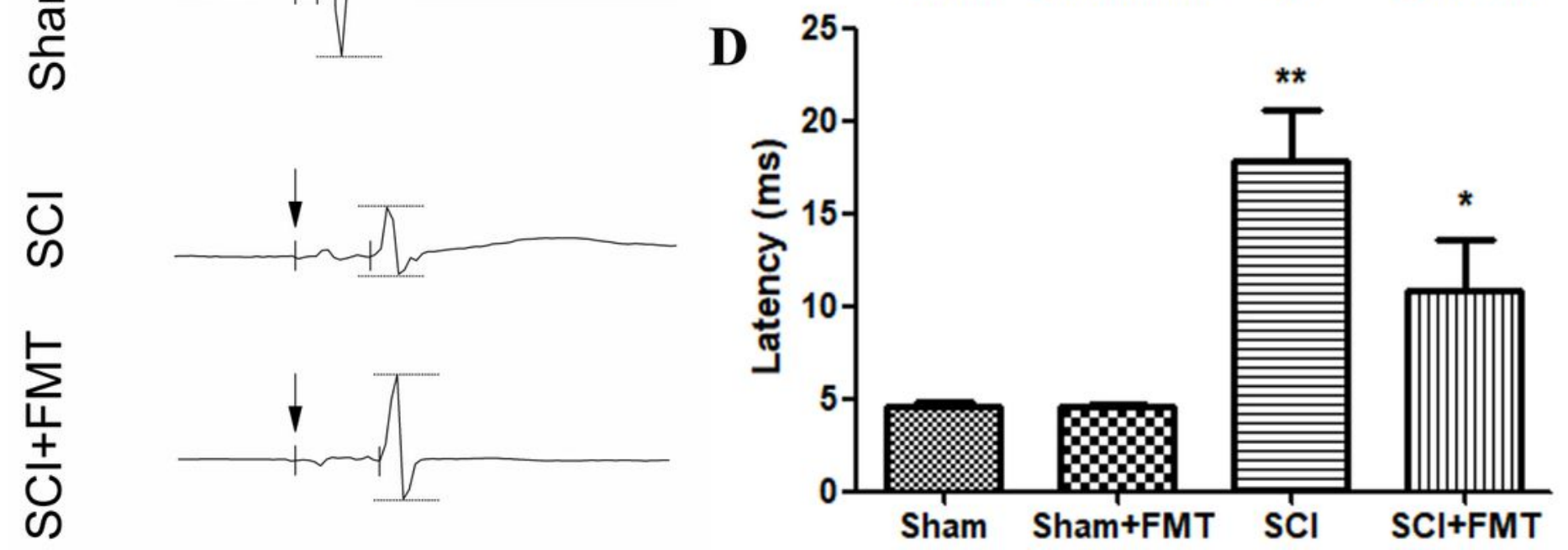

Figure 2

Effect of FMT treatment on spinal cord conduction capability. a A schematic diagram of MEP recording experiment. MEP was recorded from the gastrocnemius muscle in an anesthetized state after electrical 
stimulation on the motor cortex. b Representative MEPs recorded from Sham mice and SCI mice that had received the indicated treatment $4 \mathrm{w}$ after injury. The amplitude (c) and the latency (d) were quantified and statistically analyzed. ${ }^{\star} p<0.05$ compared to Sham group; ${ }^{*} p<0.01$ compared to Sham group; \#\#p<0.01 compared to SCl group.
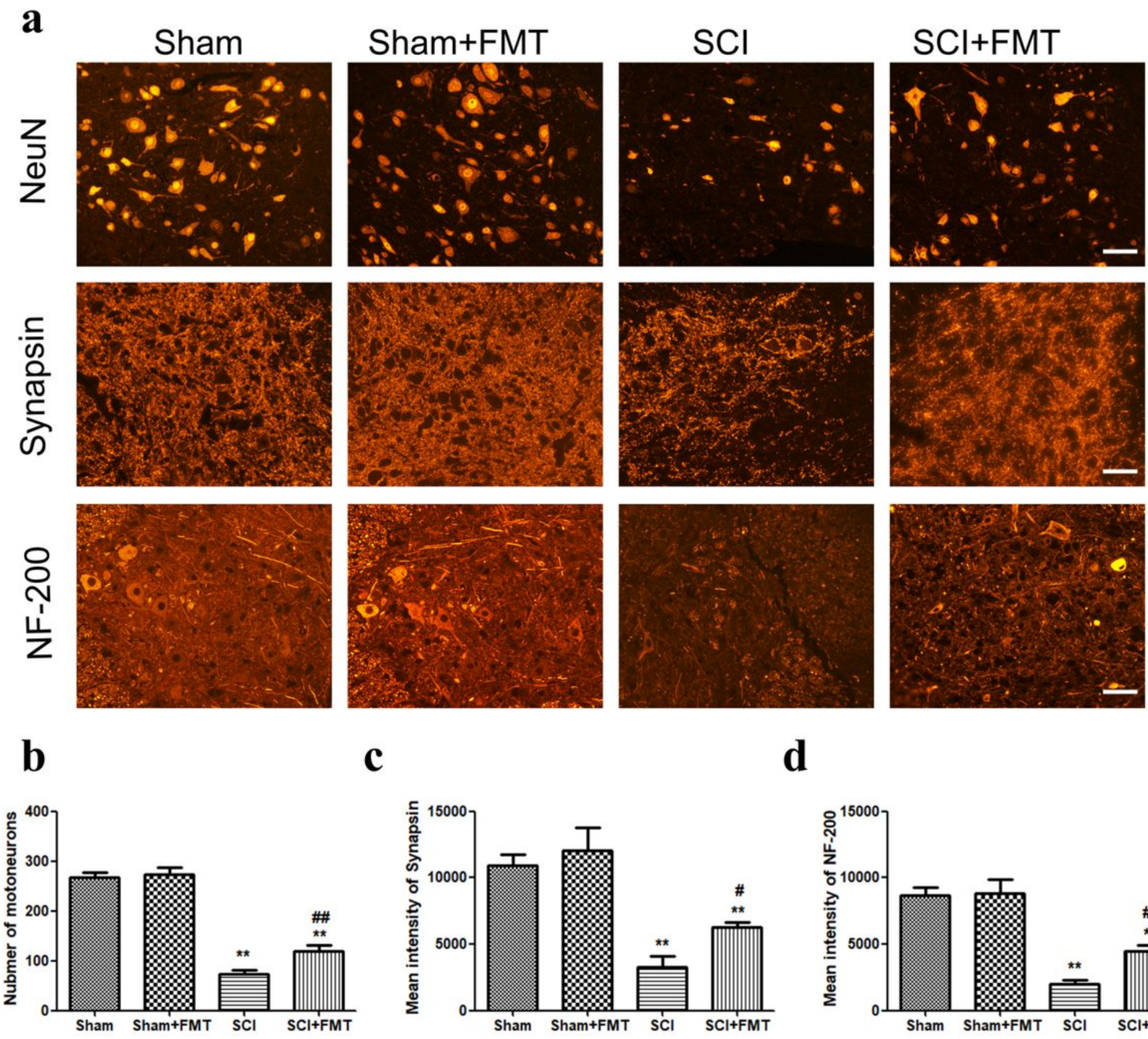

c
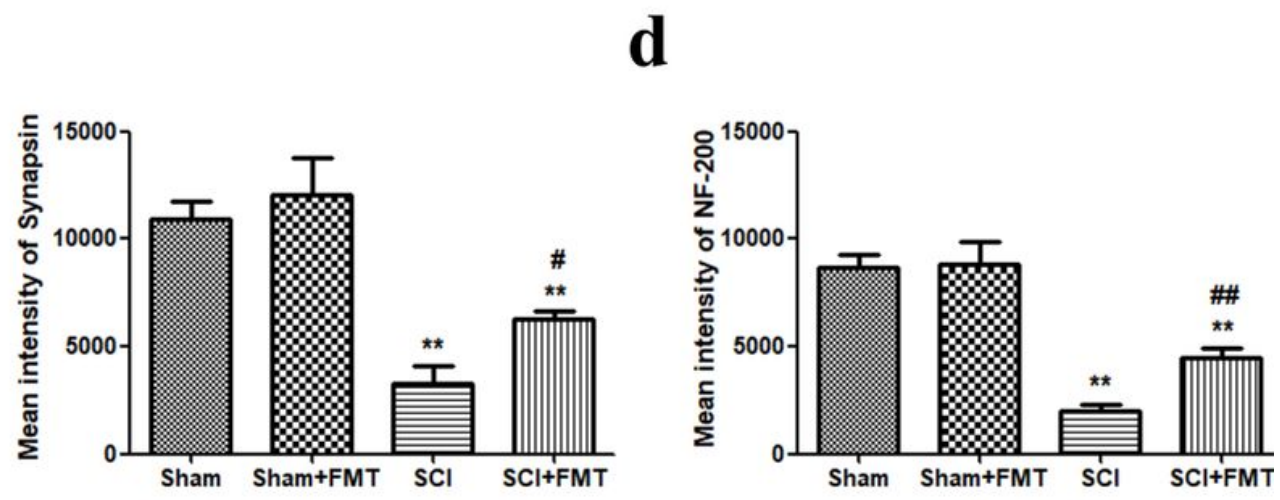

Figure 3

Effect of FMT treatment on neuronal survival and synaptic regeneration following SCl. a NeuN-positive neurons were examined in the different treatment groups by immunofluorescence staining $(n=4)$. b 
Quantification of NeuN-positive neuronal cell bodies in the T10 region in different groups. a,c Quantification of synapsin immunoreactivity (red) with representative images of the ventral horn. a,d Quantification of NF-200 immunoreactivity (red) with representative images of the ventral horn. Scale bar, $50 \mu \mathrm{m} .{ }^{*} \mathrm{p}<0.05$ compared to Sham group; ${ }^{* *} \mathrm{p}<0.01$ compared to Sham group; $\# \mathrm{p}<0.05$ compared to SCl group; \#\#p<0.01 compared to SCl group.

$\mathbf{a}$

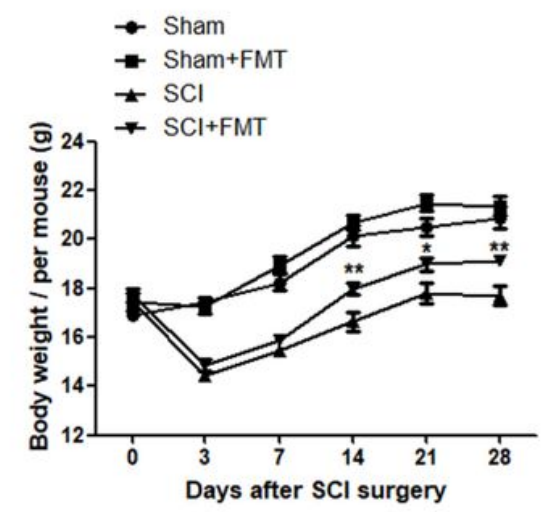

d

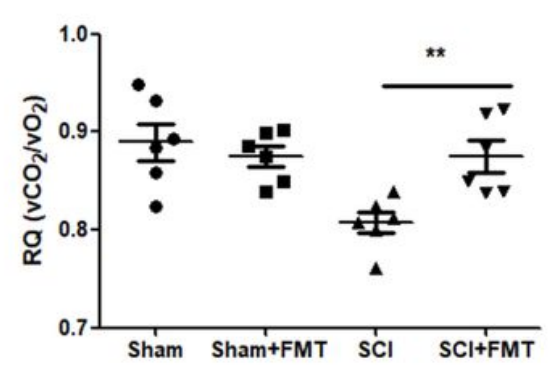

f

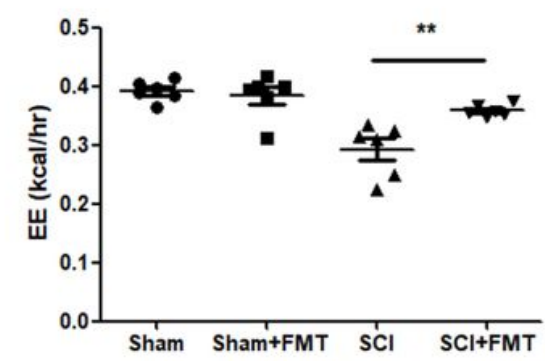

b

$$
\begin{aligned}
& \text { - SCl } \\
& \text { - SCl+FMT }
\end{aligned}
$$

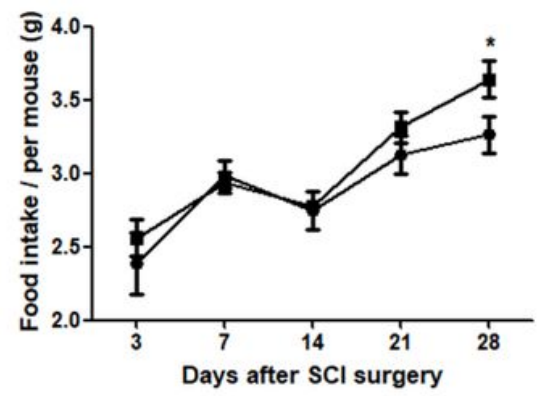

e

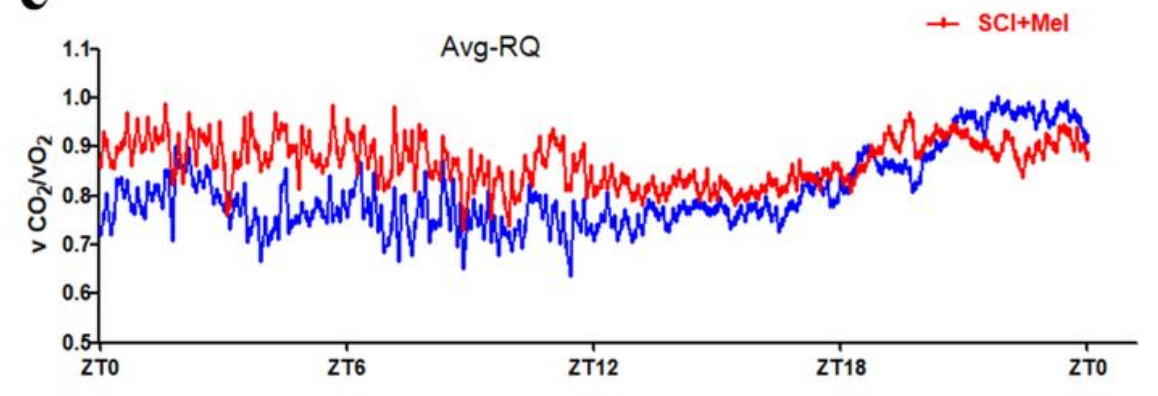

g

$+\mathrm{SCl}$

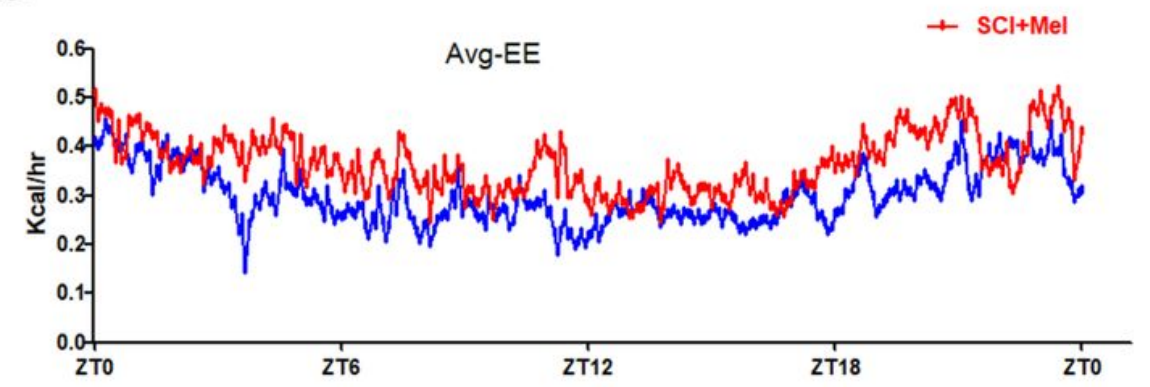

\section{Figure 4}

Effect of FMT treatment on body weight, food intake, water consumption and metabolism. a Changes in body weight over time in Sham, Sham+FMT, SCl, and SCl+FMT groups. b-c food intake (b) and water consumption (c) were examined during the 4 weeks in the four groups. Respiratory quotient (RQ) (d) and energy expenditure (EE) (f) were measured at the end of the experiments. The mean respiratory quotient 
(Avg_RQ) (e) and mean energy expenditure (Avg_EE) (g) were measured every $5 \mathrm{~min}$ for $24 \mathrm{~h}$ in $\mathrm{SCl}$ group and $\mathrm{SCl}+\mathrm{FMT}$ group. ${ }^{*} \mathrm{p}<0.05$ compared to $\mathrm{SCl}$ group; ${ }^{\star \star} \mathrm{p}<0.01$ compared to $\mathrm{SCl}$ group.

国

\section{Figure 5}

Effect of FMT treatment on intestinal permeability and expression of tight junction proteins. a Intestinal permeability was assessed 4 weeks following injury by measuring FITC intensity in serum after oral gavage of FITC-dextran. b-c Quantification of occludin immunoreactivity or ZO-1 immunoreactivity (green) with representative immunofluorescence images of colon sections. DAPI, blue; ${ }^{*} \mathrm{p}<0.05$ compared to $\mathrm{SCl}$ group; Scale bar, $50 \mu \mathrm{m} ; * \star \mathrm{p}<0.01$ compared to $\mathrm{SCl}$ group. 
a

\section{Sham Sham+FMT SCl SCl+FMT}

$2 \mathrm{H}$
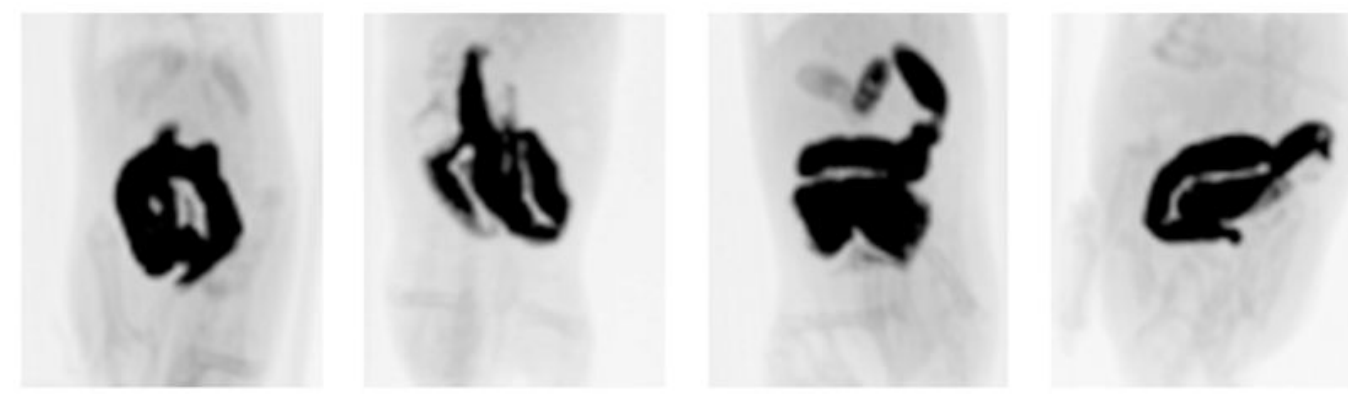

$3 \mathrm{H}$

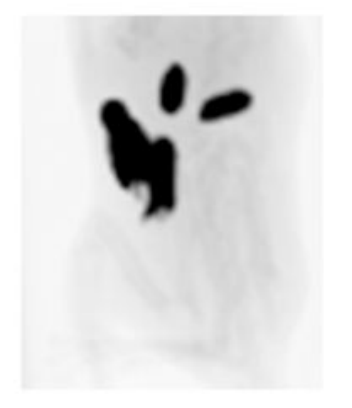

$8 \mathrm{H}$
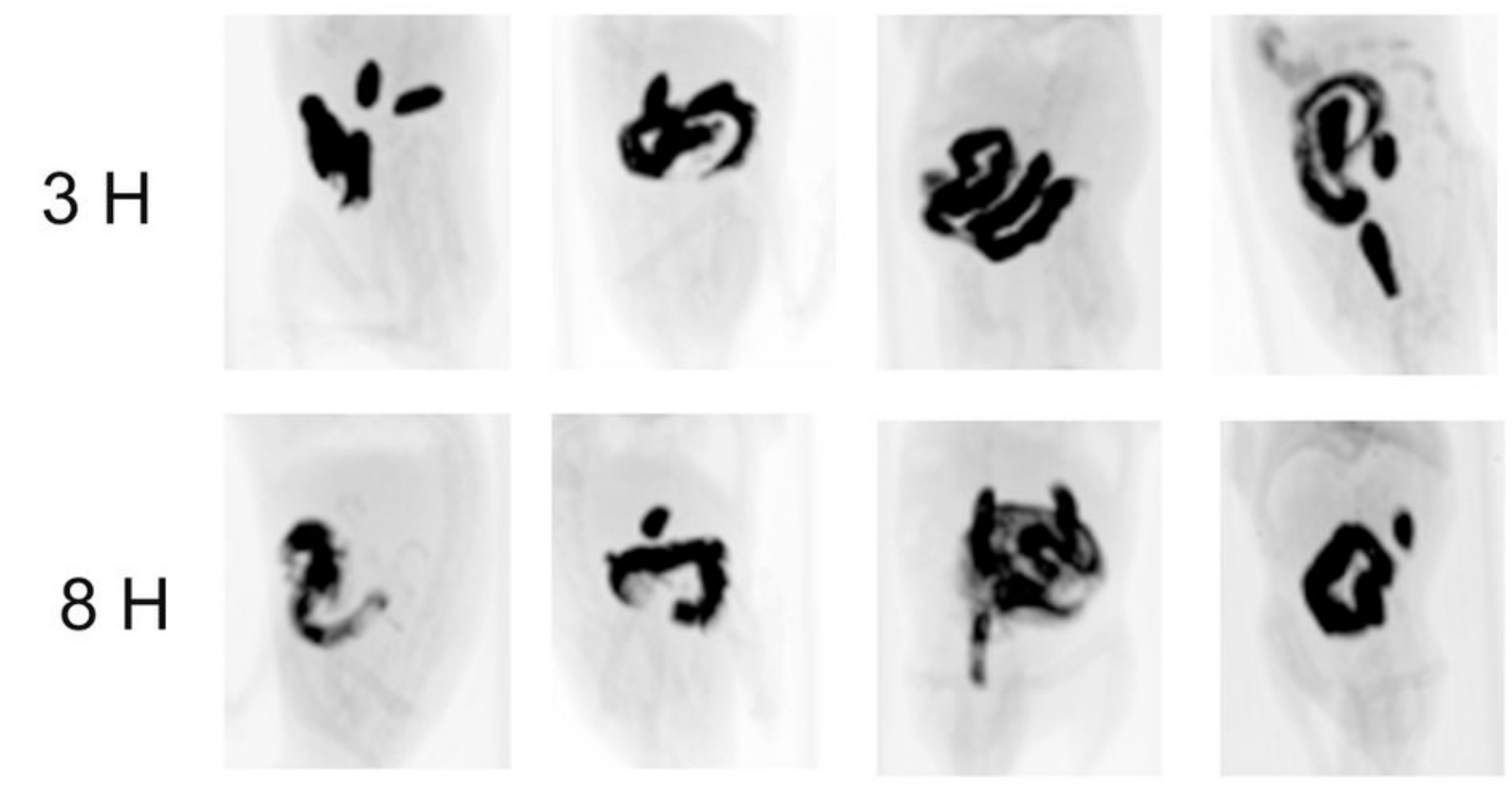

b
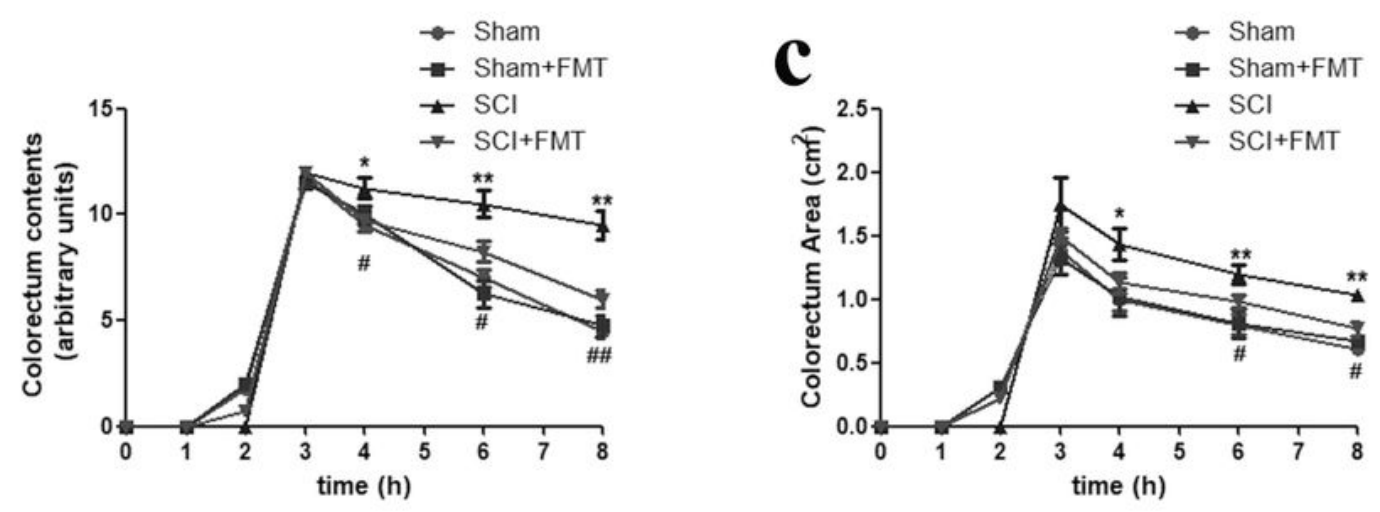

Figure 6

Effect of FMT on gastrointestinal motility in SCl mice. a Representative images of Sham, Sham+FMT, $\mathrm{SCl}$, and $\mathrm{SCl}+\mathrm{FMT}$ groups at 2, 3 and $8 \mathrm{~h}$ after administration of barium. b Filling of colorectum was measured by radiological methods. c Colorectum size was determined by using Image J. ${ }^{\star \star} \mathrm{p}<0.01$ compared to Sham group; \#p<0.05 compared to SCl group; \#\#p<0.01 compared to SCl group. 
a

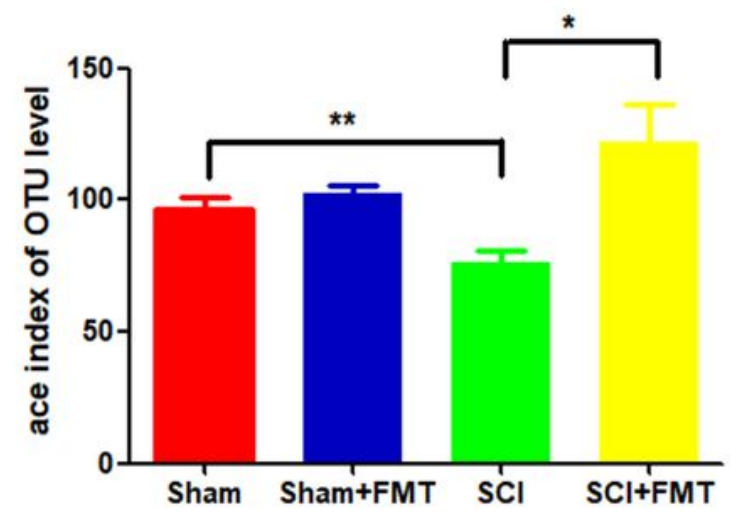

c

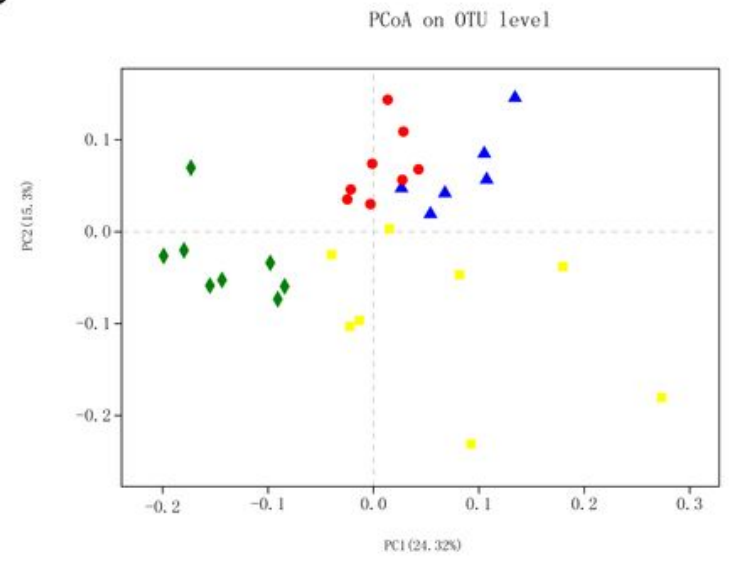

e
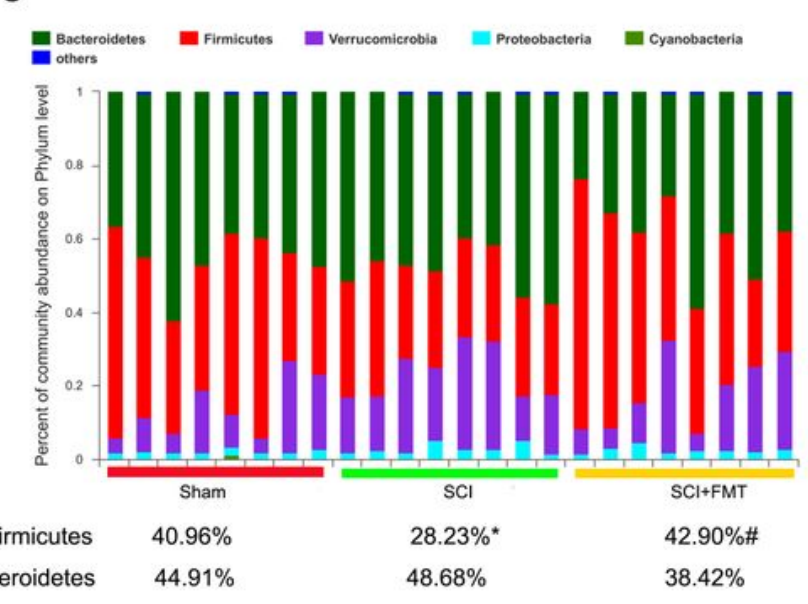

b

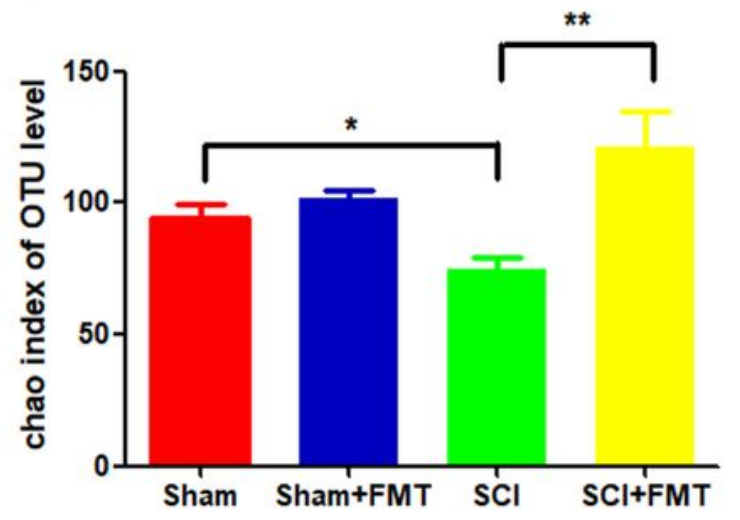

d
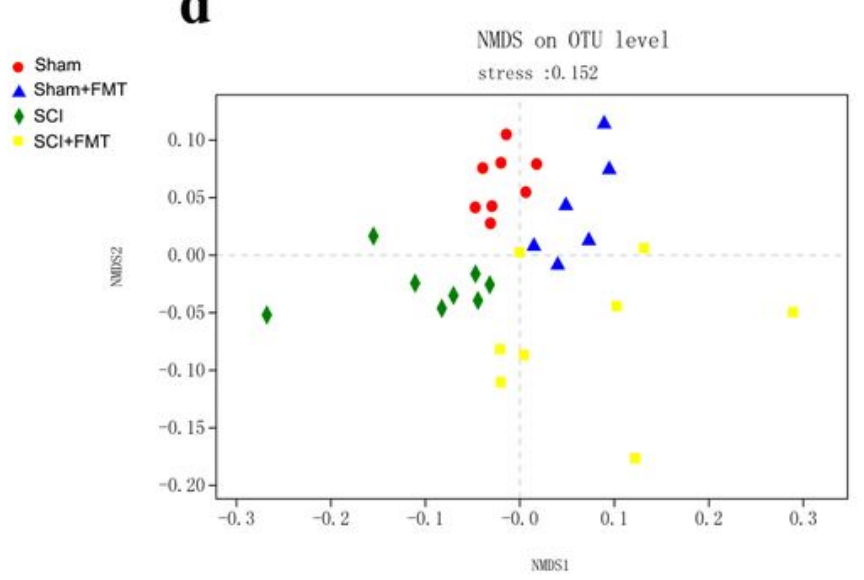

f

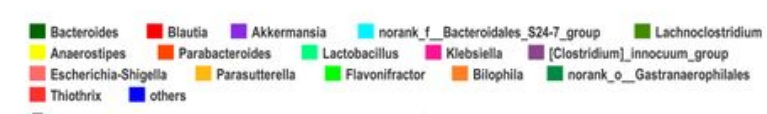

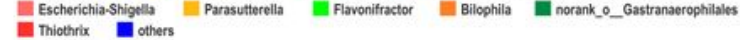

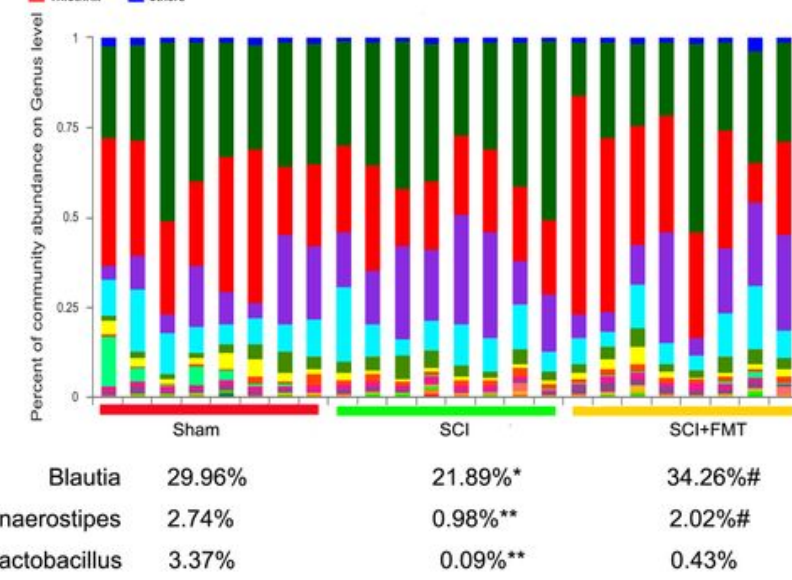

\section{Figure 7}

Effect of FMT treatment on gut bacterial composition after SCl. a-b Comparison of ace index (a) and chao index (b) based on OUT levels in the four groups. c-d Scatter plots of principal coordinate analysis (PCoA) (c) and non-metric multi-dimensional scaling (NMDS) (d) showing similarity of the bacterial communities based on uniFrac-weighted distance. (e) Bacterial composition of the different communities at phylum level and quantitative analyses of relative abundances of Firmicutes and Bacteroidetes among 
different groups. (f) Bacterial composition of different communities at genus level and quantitative analyses of the relative abundances of Blautia, Anaerostipes and Lactobacillus among different groups. ${ }^{*} \mathrm{p}<0.05$ compared to Sham group; ${ }^{* *} \mathrm{p}<0.01$ compared to Sham group; $\# \mathrm{p}<0.05$ compared to SCI group.

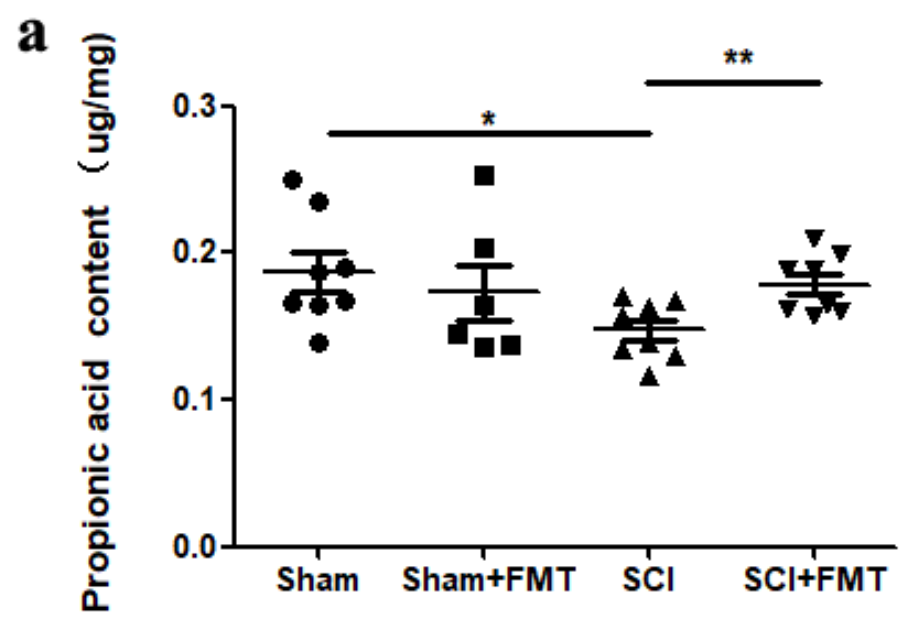

b

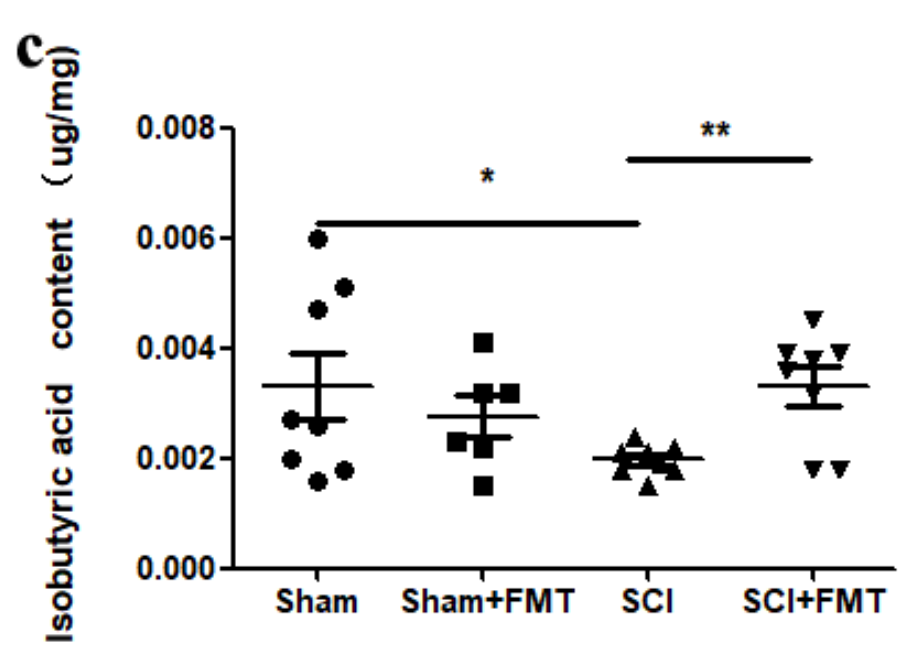

d

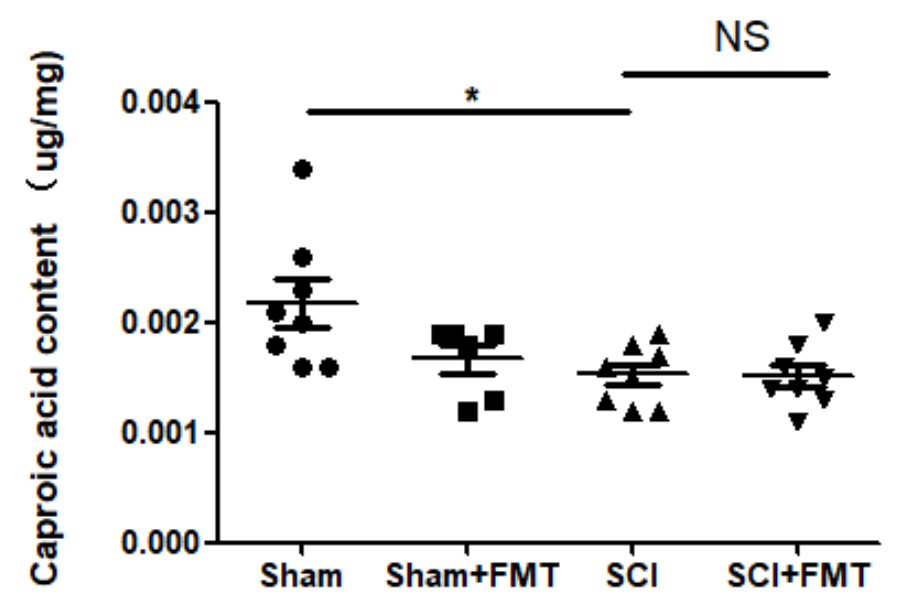

Figure 8

Effect of FMT treatment on fecal SCFA levels in mice. The SCI-mediated decrease of propionic acid, butyric acid and isobutyric acid expression was ameliorated by FMT treatment. a Fecal propionic acid content. b Fecal butyric acid content. c Fecal isobutyric acid content. The SCl-mediated decrease of caproic acid expression was not significantly changed by FMT treatment. $d$ Fecal caproic acid content. SCFAs were analyzed by GC-MS. ${ }^{*} p<0.05$ compared to SCl group; ${ }^{*} \mathrm{p}<0.01$ compared to $\mathrm{SCl}$ group; NS, not significant. 
a

$R=0.473$

$P<0.05$

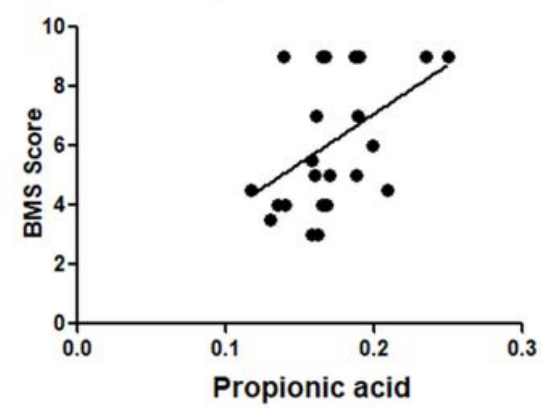

d

$R=0.574$

$P<0.01$

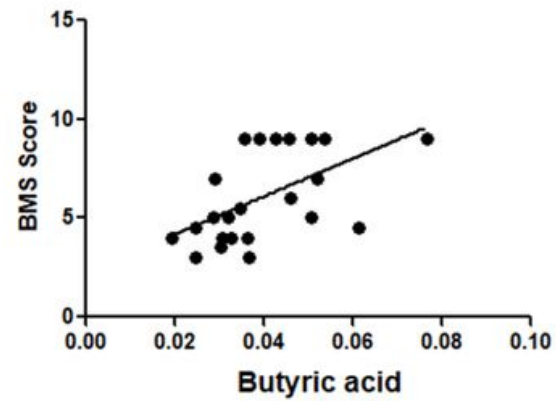

\& $\quad \begin{aligned} & R=0.272 \\ & P>0.05\end{aligned}$

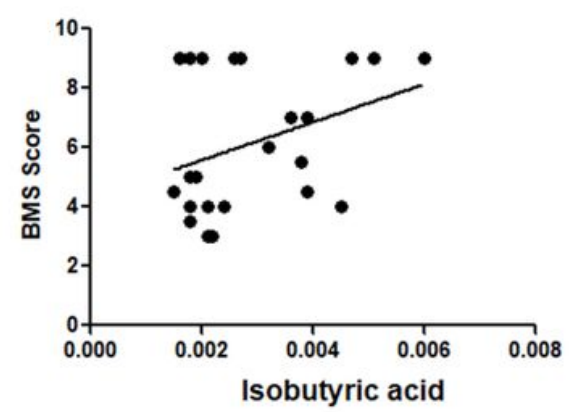

b

$$
R=0.454
$$$$
P<0.05
$$

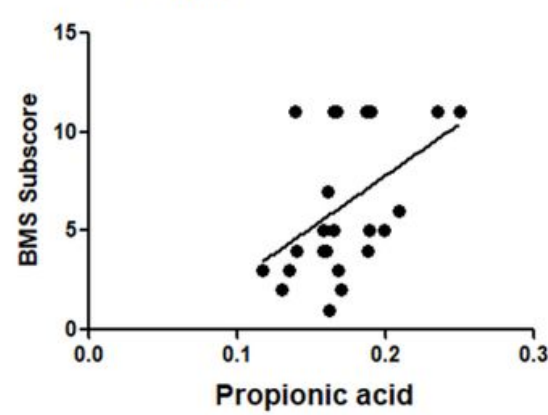

e
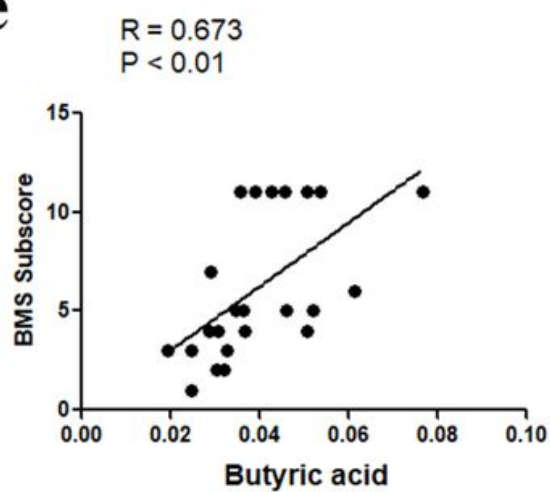

h $\quad \begin{aligned} & R=0.453 \\ & P\end{aligned}$

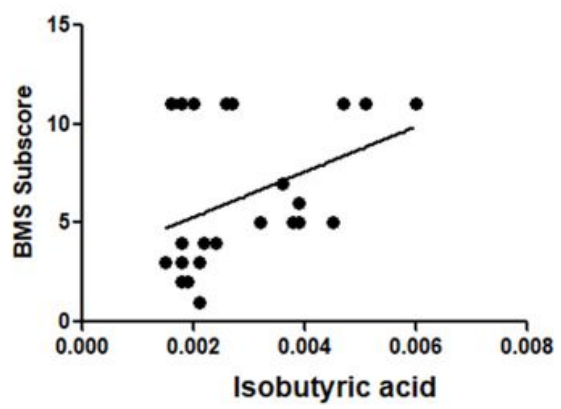

c
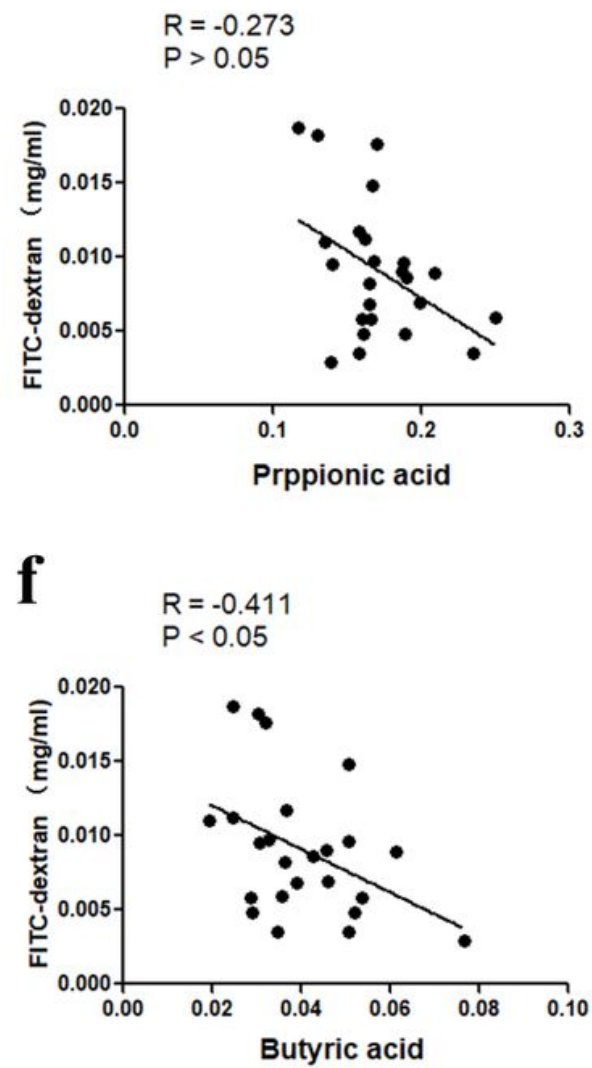

$1 \quad R=-0.387$

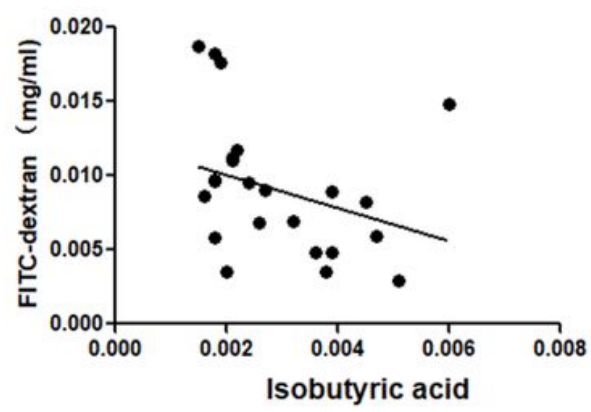

Figure 9

Correlations between SCFAs and BMS scores/BMS subscores/FITC-dextran permeability. A-c Correlations between propionic acid and BMS scores (a), BMS subscores (b), and FITC-dextran permeability (c). d-f Correlations between butyric acid and BMS scores (d), BMS subscores (e), and FITC-dextran permeability (f). h-g Correlations between isobutyric acid and BMS scores (h), BMS subscores (i), and FITC-dextran permeability $(\mathrm{g})$. 

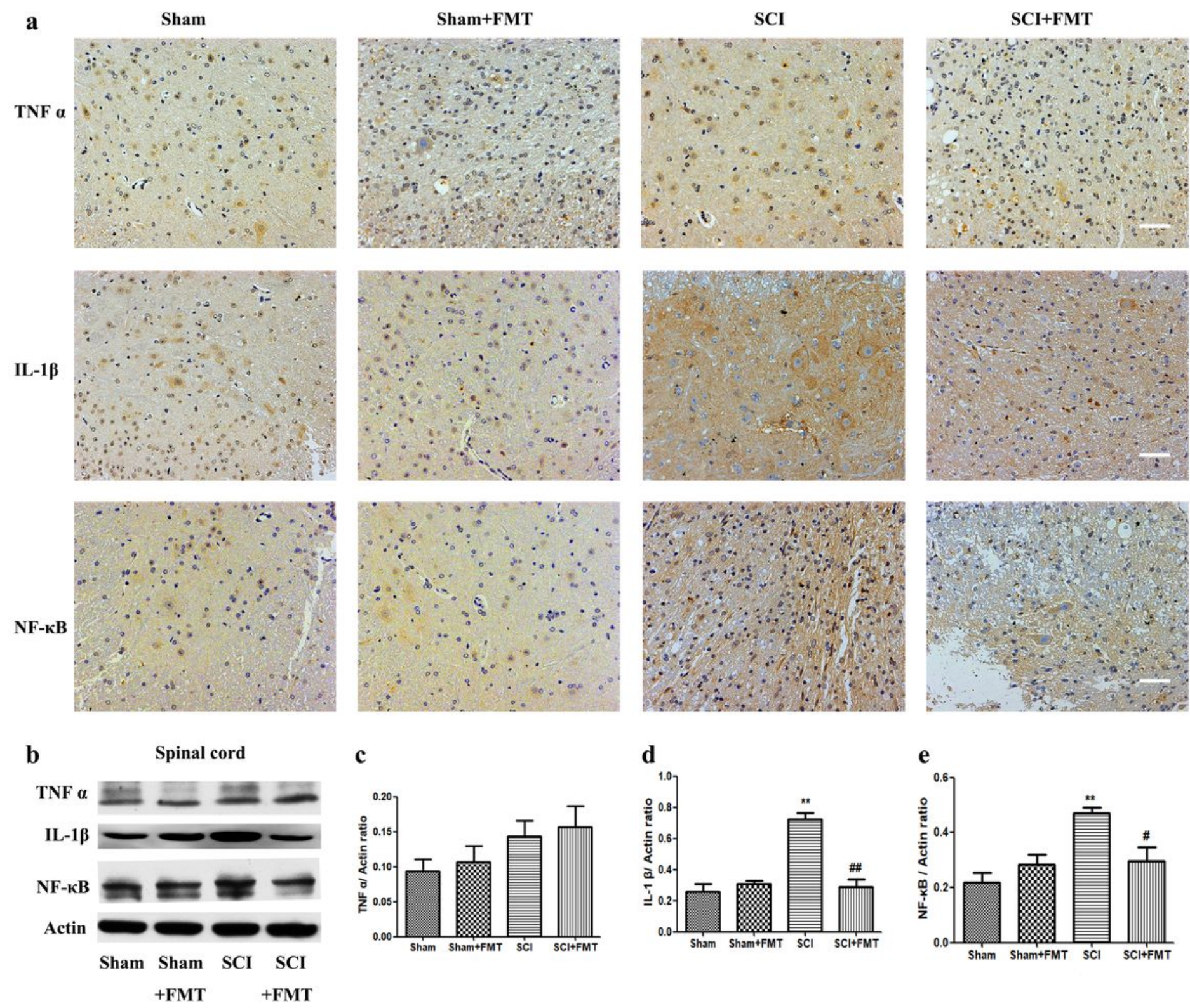

Figure 10

Effect of FMT treatment on expression of TNF $a$, IL-1 $1 \beta$ and NF-KB in spinal cord. a TNFa, IL-1 $\beta$ and NF-KB were stained by immunofluorescence on spinal cord sections from each group. Scale bar, $50 \mu \mathrm{m}$. $\mathrm{b}$ The expression of TNF $a, I L-1 \beta$ and NF-KB was detected by western blot, and the relative amount of TNFa (c), IL-1 $\beta$ (d) and NF-KB (e) were semi-quantified. 
a
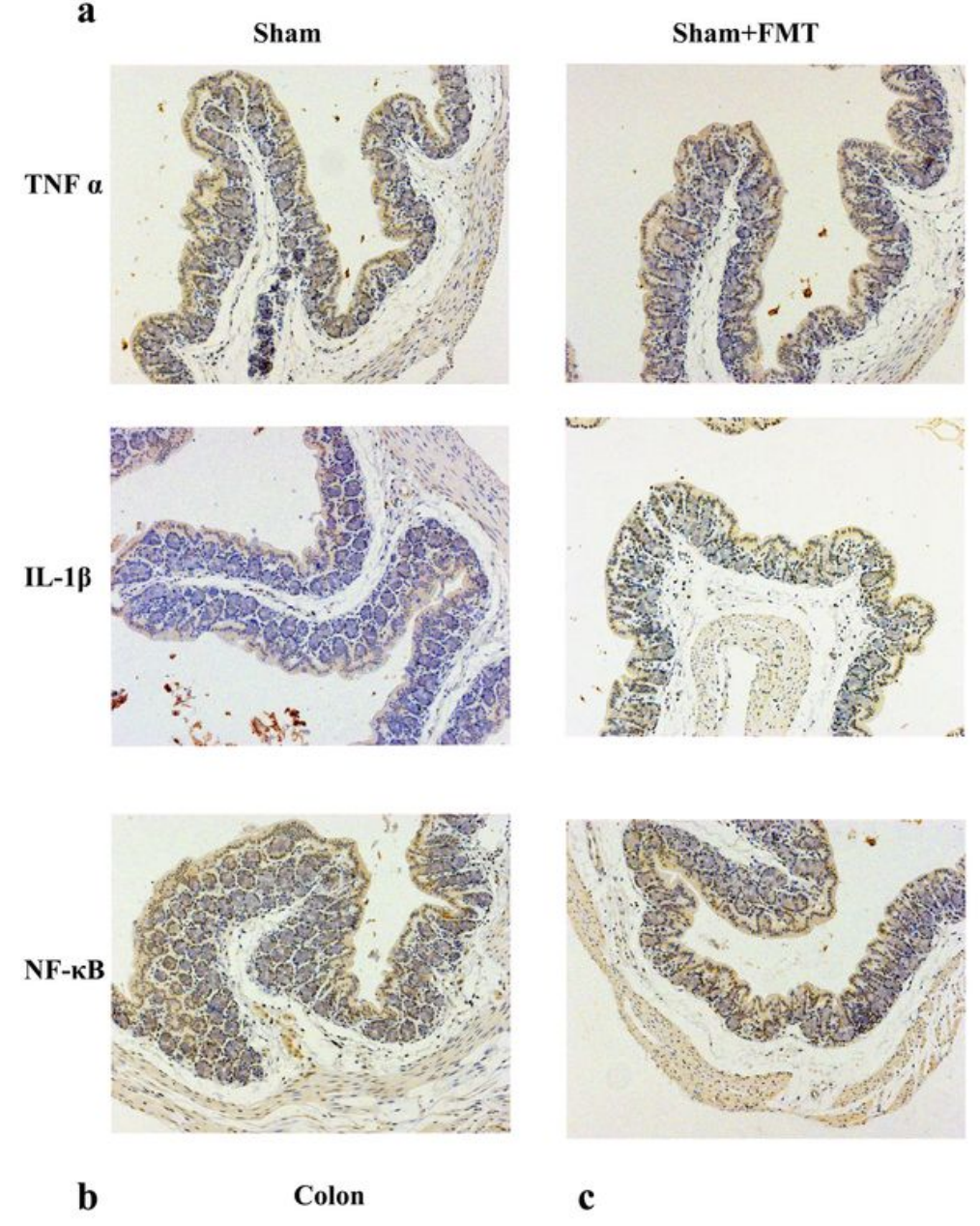

Colon
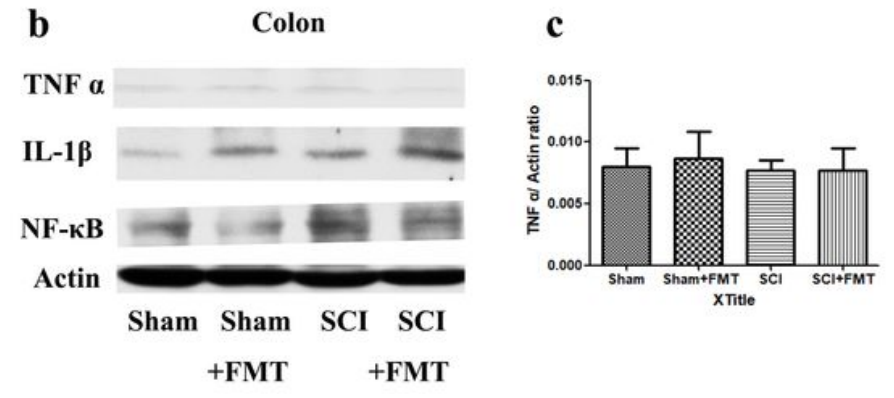
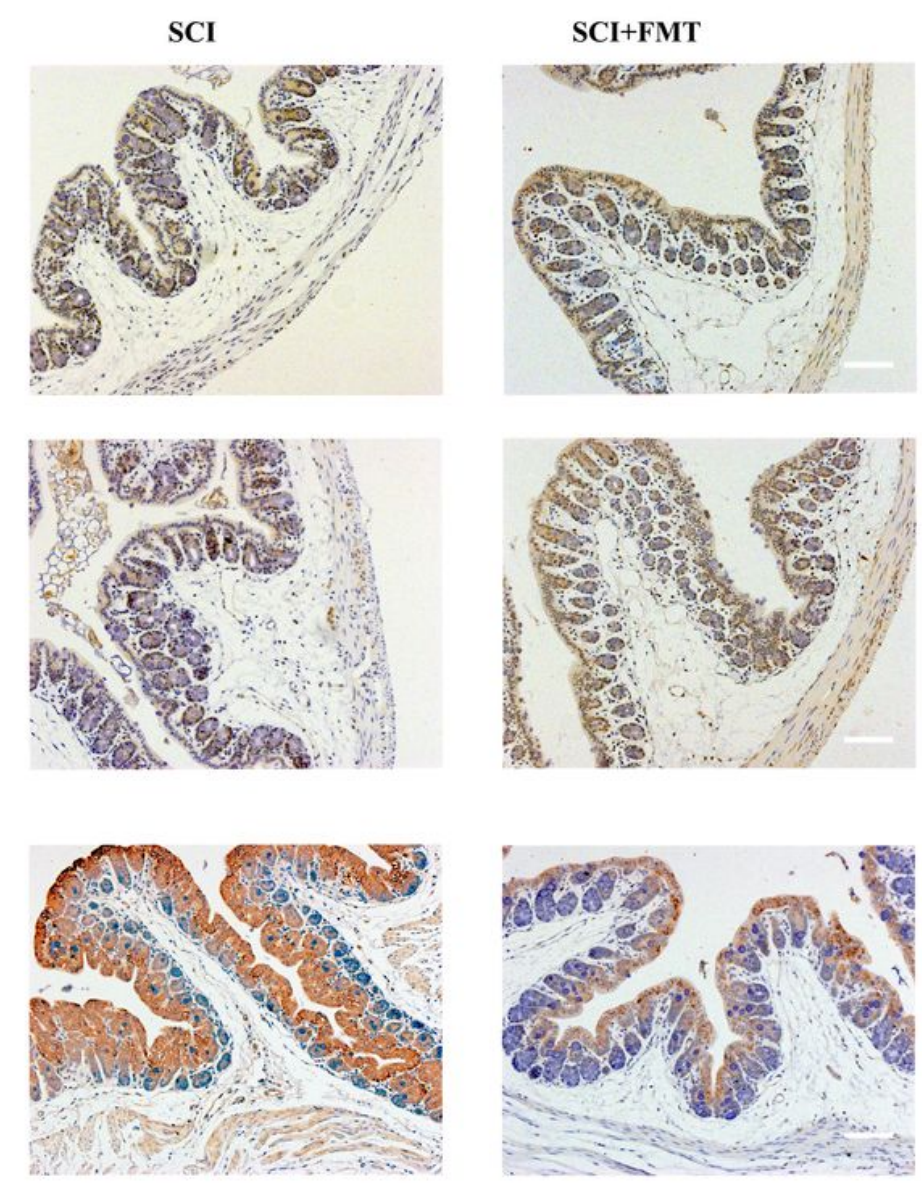

e
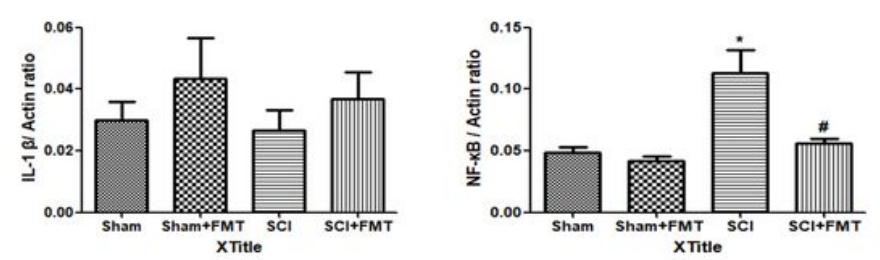

\section{Figure 11}

Effect of FMT treatment on expression of TNF a, IL-1 $\beta$ and NF-KB in colon. a TNFa, IL-1 $\beta$ and NF-KB were stained by immunofluorescence on colonic tissue from each group. Scale bar, $50 \mu \mathrm{m}$. b The expression of TNF $a, I L-1 \beta$ and NF-KB were analyzed by western blot, and the relative amount of TNF a (c), IL-1 1 (d) and NF-KB (e) were semi-quantified in the different treatment groups.

\section{Supplementary Files}

This is a list of supplementary files associated with this preprint. Click to download.

- supplementarymaterials.docx 